\title{
The Risk Management of Minimum Return Guarantees
}

Antje Mahayni ${ }^{\dagger}$, Mercator School of Management, University of Duisburg-Essen, Email: antje.mahayni@uni-due.de Erik Schlögl ${ }^{\ddagger}$, School of Finance and Economics, University of Technology, Sydney, Email: Erik.Schlogl@uts.edu.au*

\begin{abstract}
Contracts paying a guaranteed minimum rate of return and a fraction of a positive excess rate, which is specified relative to a benchmark portfolio, are closely related to unit-linked life-insurance products and can be considered as alternatives to direct investment in the underlying benchmark. They contain an embedded power option, and the key issue is the tractable and realistic hedging of this option, in order to rigorously justify valuation by arbitrage arguments and prevent the guarantees from becoming uncontrollable liabilities to the issuer. We show how to determine the contract parameters conservatively and implement robust risk-management strategies.
\end{abstract}

Keywords: Minimum return guarantee, defined-contribution pension plans, life-insurance, uncertain volatility, conservative pricing, robust hedging, model misspecification, model risk

\section{Introduction}

Many modern life-insurance policies specify minimum-rate-of-return guarantees on the capital accumulated during the life of the contract. This and other elements of optionality, such as bonus distribution schemes and surrender possibilities, implicitly represent short positions in financial derivatives and as such are liabilities, which constitute a potential hazard to company solvency. Unfortunately, up to recent times this hazard has remained unrecognised or ignored, with often catastrophic results for policy holders and insurance company shareholders alike. ${ }^{1}$ It is safe to say that the traditional actuarial methods failed to keep up with the complexity of the financial securities embedded in the insurance policies. Once the potentially disastrous consequences of mismanaging the risk associated with these implicit options became obvi-

\footnotetext{
The authors would like to thank Klaus Sandmann for fruitful discussions. The usual disclaimers apply. The research of the second author is supported under the Australian Research Council's Discovery funding scheme (project number DP0559879).

† Corresponding author, Mercator School of Management, University of Duisburg-Essen, Lotharstr. 65, 47057 Duisburg, Germany. Phone: +49-203-3792621, Fax: +49-228-3791402, Email: antje.mahayni@uni-due.de

* Phone: +61-2-9514-7785, Fax: +61-2-9514-7711

1 A set of such cases is given by Grosen and Jørgensen (1999), Briys and de Varenne (1997), Pelsser (2002) and Chen and Suchanecki (2007).
}

ous, the "fair valuation" of these liabilities became a focus of attention both in the insurance and the accounting professions. ${ }^{2}$

Minimum-rate-of-return guarantees have also begun to gain importance on another front. As aging populations increasingly put pressure on traditional defined-benefit pension plans, there is a trend toward defined-contribution plans, in order to reduce the risk borne by plan sponsors (be they government organisations or private-sector corporations). Countries such as Australia, Mexico and Chile have moved or are in the process of moving to defined-contribution plans, as are many private-sector employers in the U.S. and the U.K. This has the unfortunate side effect that, as Bodie and Crane (1999) put it, "In definedcontribution plans, investment risk is being passed on to the parties who are the least knowledgeable and the least able to bear it." It seems reasonable, as they suggest, that minimum-rate-of-return guarantees on retirement savings plans have an important role to play in addressing this problem. In order for this to be practicable, however, realistic valuation techniques and an imple-

\footnotetext{
2 This development is discussed in Jørgensen (2001), who also gives further references. See in particular the report of the Fair Valuation of Liabilities Task Force appointed by the American Academy of Actuaries, reproduced in Vanderhoof and Altman (1998).
} 
mentable risk-management methodology must be available.

Past difficulties with the financial derivatives embedded in life-insurance contracts are in part due to the fact that the classical actuarial approach, i.e. valuing liabilities by discounting expected payouts with a fixed interest rate, is not applicable. Traditionally one could justify using expected payouts by the law of large numbers, i.e. for a company with a large number of policies outstanding, the mortality tables reflect nearly deterministic proportions rather than probabilities. For claims depending on one or few underlying financial variables, this is not true. Rather, an approach based on arbitrage arguments of the type pioneered by Black and Scholes (1973) is required. Thus "fair valuation" becomes "pricing by arbitrage" where the embedded options are concerned. This was recognised early on by Brennan and Schwartz (1976, 1979) and Boyle and Schwartz (1977).

In the present paper, we consider a savings plan which yields a guaranteed interest rate plus a participation in the positive excess return of a given benchmark portfolio, in a slight modification of the insurance contract studied by Miltersen and Persson (2003). In fact, in the absence of a bonus account to smooth realised returns, our results can be mapped to their setting in a straightforward manner. It is the bonus account and the associated distribution mechanism which distinguishes what is commonly termed participating contracts from unit-linked contracts. In a pure version of the former, all return above the guaranteed level is credited to a bonus account and distributed between the policy holder and the issuer at some later date according to the contractual arrangement. We consider the latter type, where the excess return credited to the customer is linked directly to the level of some reference portfolio, such as a mutual fund (as commonly seen in practice), a certain stock, a stock index, a foreign currency, etc. As explained below, we envision a different potential role for a bonus account.

Motivated by the realisation that when unhedged and wrongly priced, the options embedded in insurance/savings products can become uncontrollable liabilities and have led to the collapse of some issuers, there has been a proliferation of recent research on this topic. Unit-linked contracts with minimum return guarantees are studied by Bacinello and Ortu (1993), Nielsen and Sand- mann (1995), Boyle and Hardy (1997), Bacinello (2001), Grosen and Jørgensen (1997), Pelsser and Schrager (2004) and Mahayni and Sandmann (2006). What is common to this strand of literature is the use of martingale pricing theory based on the works of Harrison and Kreps (1979) and Harrison and Pliska (1981). However, a great part of the existing literature is mainly concerned with the correct valuation of insurance policies, i.e. the pricing of the option component by standard Black/Scholes-type dynamic arbitrage arguments. ${ }^{3}$ The risk management of insurance products is often considered to be the concern of regulatory intervention. Thus there is a serious gap, which we aim to fill in this paper: Even if the options embedded in insurance products are priced correctly, they still represent uncontrollable liabilities if unhedged. This argument can be taken even further: the determination of "fair" prices according to standard theory is justified by the existence of hedging strategies, i.e. self-financing trading strategies which replicate the payoff of the derivative under consideration, where the fair price is to be interpreted as the initial investment in the self-financing strategy. Consequently, in this paper we seek tractable and realistic hedging strategies to justify meaningful prices for options embedded in insurance products. 4

This is not simply an extension of existing valuation results: Given the very long maturities of the products under consideration, in order to be practically meaningful, prices and hedging strategies must take into account a high degree of uncertainty about the true dynamics of the underlying asset. This "model risk" that undoubtedly exists is even more important for hedging than for pricing: While one might argue that for such long maturities, using some sort of long-run "average" volatility will result in reasonable prices, it is local (i.e. instantaneous) volatility that determines the correct hedging strategy.

\footnotetext{
3 An exception is for example the paper of Coleman, Kim, Li and Patron (2007). In contrast to our approach, the hedging strategies which are used there depend on the real world measure, i.e. do not match the definition of robustness which is used in the following.

4 In contrast, there is a strand of literature which instead takes into account the possibility of a default, see Briys and de Varenne (1994, 1997), Grosen and Jørgensen (2002), Bernard, Le Courtois and Quittard-Pinon (2005) and Chen and Suchanecki (2007).
} 
While risk management under model misspecification and model risk has received some attention in the finance literature, this has not been suitably applied to insurance products. For the purposes of the present paper, the key result from the existing literature is the observation that for convex payoffs the Black/Scholes hedging strategy is robust with respect to model misspecification if the assumed Black/Scholes volatility dominates the true volatility (and vice versa for concave payoffs). ${ }^{5}$ Building on the Uncertain Volatility Model of Avellaneda, Levy and Parás (1995) and Lyons (1995), it allows us to construct meaningful hedging strategies using a minimum of assumptions on the stochastic dynamics of the underlying asset, i.e. that the volatility is bounded above and below.

The risk-management strategy thus constructed represents a superhedge, i.e. under the assumption of uncertain but bounded volatility the payoff from the hedge dominates the liability due to the embedded option. However, the strategy is no longer self-financing in the usual sense of the word, as it will be possible to extract funds from the hedge before maturity, depending on the realised volatility of the underlying asset during the life of the option. These funds could be distributed back to the insured as they are freed by the risk-management strategy. In this sense, the risk-management of minimum return guarantees embedded in life-insurance products appears a far more practicable application of superhedging using volatility bounds than it would be for pure financial derivatives. For the latter, superhedges are typically considered too expensive to implement at a marketable price.

In other words, the present paper can also be seen as a suggestion on how to extricate pension plans from the defined-benefit/defined-contribution dilemma. The contract considered here is of the defined-contribution type, but the market risk borne by the insured is limited by the minimum return guarantee. The guarantee is risk-managed and priced using a superhedging strategy, which is robust against model misspecification, but the cost of this to the insured can be mitigated by distributing funds freed from the risk-management strategy

\footnotetext{
5 This robustness of the Black/Scholes model is discussed in detail in El Karoui, Jeanblanc-Picqué and Shreve (1998) and Dudenhausen, Schlögl and Schlögl (1998). It was first observed by Avellaneda, Levy and Parás (1995).
}

during the life of the contract using a bonus distribution mechanism not unlike those currently common in life insurance.

The rest of the paper is organised as follows. Section 2 defines the payoff structure of a rollover savings plan, which can be interpreted as a life-insurance product which pays out a minimum rate of return $g$ and a fraction $\alpha$ of a positive excess which is specified on the basis of a benchmark portfolio. It is shown that the option implicit in the guarantee can be expressed as a suitably specified power option, the valuation of which in a Black/Scholes-type financial market model is well-known in the finance literature. These results are used to determine the "fair" contract parameters $(\alpha, g)$ such that no additional upfront premium is necessary. Section 3 is concerned with model risk. We give a short review of well-known results which are needed in order to achieve the conservative contract specification and robust hedging strategy, including the definition of the cost process for imperfect hedges. Static hedges using standard options are discussed in Section 4. These hedges are completely modelindependent and thus unaffected by model misspecification. In addition to giving useful intuition for the dynamic strategies, the static hedges allow us to imply bounds on the fair contract parameters from market-quoted option prices. However, the standard options are typically unavailable for very long maturities, so Section 5 considers conservative contract specifications arising from dynamic robust hedging strategies. The cost process of the superhedge then describes how funds are freed from the risk-management strategy during the life of the contract. We conclude with some final remarks.

\section{The Embedded Power Option}

Along the lines of Miltersen and Persson (2003), we assume that if the insured pays $K_{i}$ at each time $t_{i}$, the payoff at maturity $t_{N}$ is ${ }^{6}$

$$
I\left(t_{N}\right)=\sum_{i=0}^{N-1} K_{i} e^{g_{i}\left(t_{N}-t_{i}\right)+\alpha\left[\delta\left(t_{i}, t_{N}\right)-g_{i}\left(t_{N}-t_{i}\right)\right]^{+}}
$$

\footnotetext{
$6 t_{N}$ can be interpreted as the "time of retirement", when premium payments cease and in the simplest case the accumulated funds are paid out as a lump sum.
} 
where the + superscript denotes the positive part of $\delta\left(t_{i}, t_{N}\right)-g_{i}\left(t_{N}-t_{i}\right)$, i.e. $\max \left(0, \delta\left(t_{i}, t_{N}\right)-g_{i}\left(t_{N}-t_{i}\right)\right)$. This is in effect a roll-over savings plan in which all dividends and interest earned are reinvested: At maturity $t_{N}$ the insured receives his insurance premium plus a guaranteed minimum rate of return $g$ and a fraction $\alpha$ of the positive excess rate of return, if any, calculated with respect to a benchmark index $X$, i.e.

$$
\delta\left(t_{i}, t_{N}\right):=\ln \frac{X_{t_{N}}}{X_{t_{i}}}
$$

Thus a return is guaranteed on each premium payment individually, while still realistically modelling the accumulation of funds during the life of the contract. ${ }^{7}$ Consider the payoff which is directly associated with the premium $K_{i}$, i.e.

(3) $\quad I^{(i)}\left(t_{N}\right):=K_{i} e^{g_{i}\left(t_{N}-t_{i}\right)+\alpha\left[\delta\left(t_{i}, t_{N}\right)-g_{i}\left(t_{N}-t_{i}\right)\right]^{+}}$.

It is immediately clear that the payoff function is increasing with respect to the participation rate $\alpha$, while the effect of changing the guaranteed rate $g$ is twofold: Increasing $g$ raises the level of the guaranteed payoff and the point at which the insured begins to participate in gains of the benchmark index. This is easily seen when notation is adjusted to decompose the payoff into a guaranteed part and an embedded option.

Lemma 2.1 For $\delta\left(t_{i}, t_{N}\right)=\ln \frac{X_{t_{N}}}{X_{t_{i}}}$ it holds

$$
\begin{aligned}
I^{(i)}\left(t_{N}\right)= & \frac{K_{i}}{G\left(t_{i}, t_{N}\right)} \\
& +N^{(i)}\left(\alpha, g_{i}\right)\left[X_{t_{N}}^{\alpha}-K^{(i)}\left(\alpha, g_{i}\right)\right]^{+}
\end{aligned}
$$

where

$$
\begin{aligned}
G\left(t_{i}, t_{N}\right) & :=\exp \left\{-g_{i}\left(t_{N}-t_{i}\right)\right\}, \\
N^{(i)}\left(\alpha, g_{i}\right) & :=K_{i} \frac{G^{\alpha-1}\left(t_{i}, t_{N}\right)}{X_{t_{i}}^{\alpha}} \\
K^{(i)}\left(\alpha, g_{i}\right) & :=\left(\frac{G\left(t_{i}, t_{N}\right)}{X_{t_{i}}}\right)^{-\alpha}
\end{aligned}
$$

7 Miltersen and Persson (2003) consider a contract which guarantees a minimum return for each individual year of the life of the contract, i.e. ceteris paribus their guarantee dominates the one given here. However, in keeping with the interpretation that the contact represents savings toward retirement, we consider the insured to be only concerned about the return over the total life of the contract, and the specification given here is the less expensive version of the guarantee. For the analysis below, the results for both specifications are completely analogous.

\section{Proof. See Appendix A.}

With the above lemma it follows that the fair valuation of the MRRG (Minimum Rate of Return Guarantee) is given by the present value of the fixed amount plus the fair valuation of the embedded power options, i.e. for $t_{i} \leq t \leq t_{N}$ we have

$$
\begin{aligned}
& \operatorname{MRRG}^{(i)}\left(t, t_{N}\right)=\operatorname{PV}\left(\frac{K_{i}}{G\left(t_{i}, t_{N}\right)}\right) \\
& \quad+N^{(i)}\left(\alpha, g_{i}\right) P O\left(\alpha, t, t_{N}, K^{(i)}\left(\alpha, g_{i}\right)\right)
\end{aligned}
$$

where $\mathrm{MRRG}^{(i)}\left(t, t_{N}\right)$ denotes the $t$-price associated with the payoff $I^{(i)}\left(t_{N}\right), P V$ the present value operator (for deterministic cashflows) and $P O\left(\alpha, t, t_{N}, K\right)$ the price of a power option with power $\alpha$, strike $K$ and maturity $t_{N}$ on the index $X$. In the following, we use standard theory from financial economics, which is based on arbitrage arguments, to calculate the fair price of a power option.

Denote by $B(t, T)$ the time $t$ price of a zero-coupon bond paying one monetary unit at maturity $T$. The forward price process of the index $X$ is modelled as a lognormal martingale under the $t_{N}$ forward measure $\mathbf{P}_{t_{N}}:{ }^{8}$

$$
d \frac{X(t)}{B\left(t, t_{N}\right)}=\frac{X(t)}{B\left(t, t_{N}\right)} \sigma\left(t, t_{N}\right) d W_{t_{N}}(t) .
$$

This removes the need to assume deterministic interest rates, which would be unrealistic given the long time horizon. $\sigma\left(t, t_{N}\right)$ is the volatility of the forward price process and for tractability reasons we assume this volatility to be deterministic. ${ }^{9}$ One (but not the only) modelling choice which supports this would be to have $X$ follow a geometric Brownian motion (i.e. a dynamic along the lines of Black and Scholes (1973)) and interest rate dynamics given by a Gauss-Markov Heath, Jarrow and Morton (1992) model.

\footnotetext{
8 This is the martingale measure associated with taking the zero-coupon bond maturing in $t_{N}$ as the numeraire. For the relationship between different numeraires and their associated martingale measures, see Geman, El Karoui and Rochet (1995). 9 Note that this is the only assumption (and one that will be relaxed below). The fact that (6) is driftless under $\mathbf{P}_{t_{N}}$ follows from the no-arbitrage conditions. Furthermore, the calculations presented below are valid in the general multifactor case, where $W_{t_{N}}$ is a $d$-dimensional Brownian motion under $\mathbf{P}_{t_{N}}$ and $\sigma\left(t, t_{N}\right)$ is a deterministic, vector-valued function, with all products between vectors interpreted as scalar ("dot”) products.
} 
Theorem 2.2 Consider an option on the asset $X$ where the payoff at maturity $T=t_{N}$ is given by $\left[X_{T}^{\alpha}-K\right]^{+}$, i.e. an asymmetric call power option with power $\alpha$ and strike $K$. In a model where the forward index process $X$ is a lognormal martingale under the $t_{N}$ forward measure $\mathbf{P}_{t_{N}}$, as in Equation (6), it holds that

(a) The price process $P O=\left(P O_{t}\right)_{0 \leq t \leq T}$ of the power option is given by

\section{(7) $\quad P O\left(t, X_{t}\right)$}

$$
\begin{aligned}
= & B\left(t, t_{N}\right) \times\left[\left(\frac{X_{t}}{B\left(t, t_{N}\right)}\right)^{\alpha} e^{-\frac{1}{2} \alpha(1-\alpha) v_{t_{N}}^{2}(t)}\right. \\
& \times \mathcal{N}\left(h_{1}\left(t, \frac{X_{t}}{B\left(t, t_{N}\right) \sqrt[\alpha]{K}}\right)-(1-\alpha) v_{t_{N}}(t)\right) \\
& \left.-K \mathcal{N}\left(h_{2}\left(t, \frac{X_{t}}{B\left(t, t_{N}\right) \sqrt[\alpha]{K}}\right)\right)\right]
\end{aligned}
$$

where $\mathcal{N}$ denotes the one-dimensional standard normal distribution function, $\sigma\left(., t_{N}\right)$ is the deterministic volatility of the forward index, and the functions $h_{1,2}$ are given by

$$
\begin{aligned}
& h_{1}(t, z)=\frac{\ln z+\frac{1}{2} v_{t_{N}}^{2}(t)}{v_{t_{N}}(t)} ; \\
& h_{2}(t, z)=h_{1}(t, z)-v_{t_{N}}(t)
\end{aligned}
$$

and

$$
v_{t_{N}}(t)=\sqrt{\int_{t}^{t_{N}} \sigma^{2}\left(s, t_{N}\right) d s .}
$$

(b) The delta of the option is given by

$$
\text { (8) } \Delta^{P O}\left(t, X_{t}\right)=P O_{x}\left(t, X_{t}\right)
$$

$$
\begin{aligned}
= & \alpha\left(\frac{X_{t}}{B\left(t, t_{N}\right)}\right)^{\alpha-1} e^{-\frac{1}{2} \alpha(1-\alpha) v_{t_{N}}^{2}(t)} \\
& \times \mathcal{N}\left(h_{1}\left(t, \frac{X_{t}}{B\left(t, t_{N}\right) \sqrt[\alpha]{K}}\right)-(1-\alpha) v_{t_{N}}(t)\right) .
\end{aligned}
$$

(c) The gamma of the option is given by

(9) $\gamma^{P O}\left(t, X_{t}\right)=P O_{x x}\left(t, X_{t}\right)$

$$
\begin{aligned}
& =\alpha \frac{1}{B\left(t, t_{N}\right)}\left(\frac{X_{t}}{B\left(t, t_{N}\right)}\right)^{\alpha-2} e^{-\frac{1}{2} \alpha(1-\alpha) v_{t_{N}}^{2}(t)} \\
& \times\left[(\alpha-1) \mathcal{N}\left(h_{1}\left(t, \frac{X_{t}}{B\left(t, t_{N}\right) \sqrt[\alpha]{K}}\right)-(1-\alpha) v_{t_{N}}(t)\right)\right. \\
& \left.+\frac{\mathcal{N}^{\prime}\left(h_{1}\left(t, \frac{X_{t}}{B\left(t, t_{N}\right) \sqrt[\alpha]{K}}\right)-(1-\alpha) v_{t_{N}}(t)\right)}{v_{t_{N}}(t)}\right] .
\end{aligned}
$$

(d) The vega of the option is given by

$$
\begin{aligned}
\text { (10) } & P O_{v_{t_{N}}}\left(t, X_{t}\right)=v_{t_{N}}(t) X_{t}^{2} \gamma^{P O}\left(t, X_{t}\right) \\
\Leftrightarrow & P O_{\bar{\sigma}_{t_{N}}}=\bar{\sigma}_{t_{N}}(t)\left(t_{N}-t\right) X_{t}^{2} \gamma^{P O}\left(t, X_{t}\right)
\end{aligned}
$$

where $\bar{\sigma}_{t_{N}}$ is the per annum average volatility over the life of the option.

(e) The theta of the option is given by

$$
\text { (11) } \begin{aligned}
\theta^{P O}\left(t, X_{t}\right)= & P O_{t}\left(t, X_{t}\right) \\
= & r P O\left(t, X_{t}\right) \\
& +v_{t_{N}}^{\prime}(t) v_{t_{N}}(t) X_{t}^{2} \gamma^{P O}\left(t, X_{t}\right)
\end{aligned}
$$

where $\mathrm{PO}_{t}, \mathrm{PO}_{x}, \mathrm{PO}_{x x}$ denote the partial derivatives.

Proof. The above theorem is a well-known result, see for example Zhang (1998), p. 597, Equation (30.3). The proof is easily done by using a change of measure, as demonstrated in Esser (2003), and given in Appendix B for the reader's convenience. The partial derivatives are derived in Appendix C with respect to the forward prices, i.e.

$\begin{aligned} \text { (12) } P O^{*}\left(t, X_{t}^{*}\right) & =\frac{P O\left(t, X_{t}\right)}{B(t, T)}, \\ \text { where } X_{t}^{*} & :=\frac{X_{t}}{B(t, T)} .\end{aligned}$

In particular, this implies

$$
\begin{aligned}
P O_{x}\left(t, X_{t}\right)= & P O_{x}^{*}\left(t, X_{t}^{*}\right) \\
P O_{x x}\left(t, X_{t}\right)= & \frac{1}{B(t, T)} P O_{x x}^{*}\left(t, X_{t}^{*}\right) \\
P O_{t}\left(t, X_{t}\right)= & B_{t}(t, T) P O^{*}\left(t, X_{t}^{*}\right) \\
& +B(t, T) P O_{t}^{*}\left(t, X_{t}^{*}\right) \\
= & r_{t} P O\left(t, X_{t}\right)+B(t, T) P O_{t}^{*}\left(t, X_{t}^{*}\right)
\end{aligned}
$$

where $r_{t}$ is the continuously compounded short interest rate at time $t$.

Proposition 2.3 In a model where the forward index process $X$ is a lognormal martingale under the $t_{N}$ forward measure $\mathbf{P}_{t_{N}}$, as in Equation (6), it holds that the arbitrage free ("fair") price at time $t,\left(t_{i}<t \leq t_{N}\right)$, for the $M R R G^{(i)}$ on the premium $K_{i}$ 


\section{Fair Contract Parameter Combinations}

Figure 1: Short maturity $(T=1, r=0.1, \sigma=0.1, \sigma=0.2$ (dashed line), $\sigma=0.4$ (thick line))

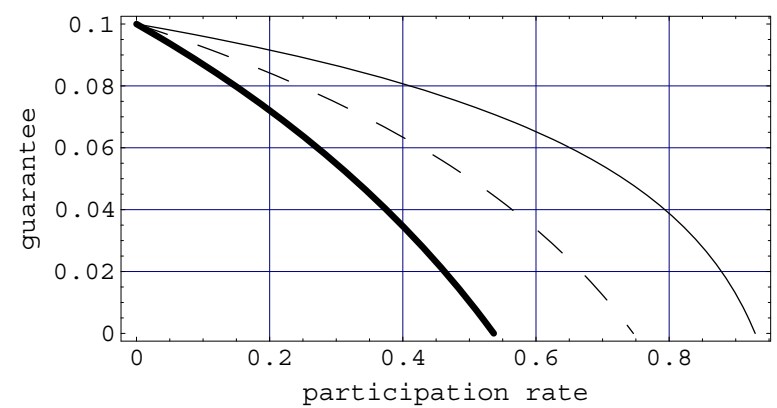

Figure 3: Short maturity $(T=1, r=0.08, g=0, g=0.02$ (dashed line))

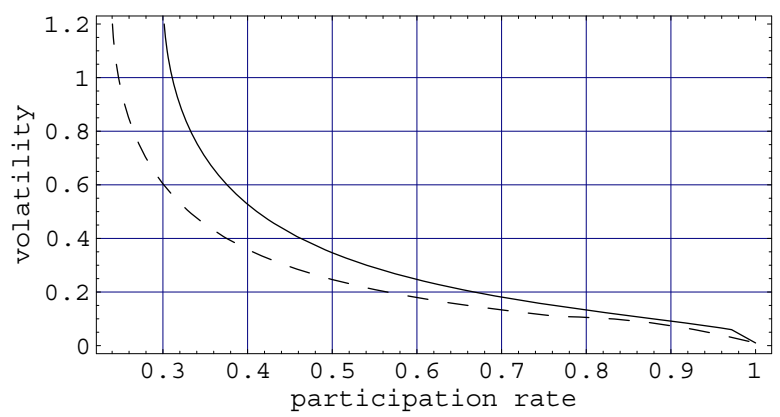

is given by

(16) $\operatorname{MRRG}^{(i)}\left(t, t_{N}\right)=\frac{K_{i} B\left(t, t_{N}\right)}{G\left(t_{i}, t_{N}\right)}$

$$
\begin{aligned}
& \times\left[1+\left(\frac{\frac{X_{t}}{X_{t_{i}}}}{\frac{B\left(t, t_{N}\right)}{G\left(t_{i}, t_{N}\right)}}\right)^{\alpha} e^{-\frac{1}{2} \alpha(1-\alpha) v_{t_{N}}^{2}(t)}\right. \\
& \times \mathcal{N}\left(h_{1}^{(i)}\left(t, \frac{\frac{X_{t}}{X_{t_{i}}}}{\frac{B\left(t, t_{N}\right)}{G\left(t_{i}, t_{N}\right)}}\right)-(1-\alpha) v_{t_{N}}(t)\right) \\
& \left.-\mathcal{N}\left(h_{2}^{(i)}\left(t, \frac{\frac{X_{t}}{X_{t_{i}}}}{\frac{B\left(t, t_{N}\right)}{G\left(t_{i}, t_{N}\right)}}\right)\right)\right]
\end{aligned}
$$

where

$$
\begin{aligned}
h^{(1)}(t, z) & =\frac{\ln z+\frac{1}{2} v_{t_{N}}^{2}(t)}{v_{t_{N}}(t)}, \\
h^{(2)}(t, z) & =h^{(1)}(t, z)-v_{t_{N}}(t), \\
v_{t_{N}}(t) & =\sqrt{\int_{t}^{t_{N}} \sigma^{2}\left(s, t_{N}\right) d s .}
\end{aligned}
$$

Proof. See Appendix D.

It is worth mentioning that, without introducing an additional upfront premium, the set of fair contract
Figure 2: Long maturity $(T=30, r=0.1, \sigma=0.1, \sigma=0.2$ (dashed line), $\sigma=0.8$ (thick line))

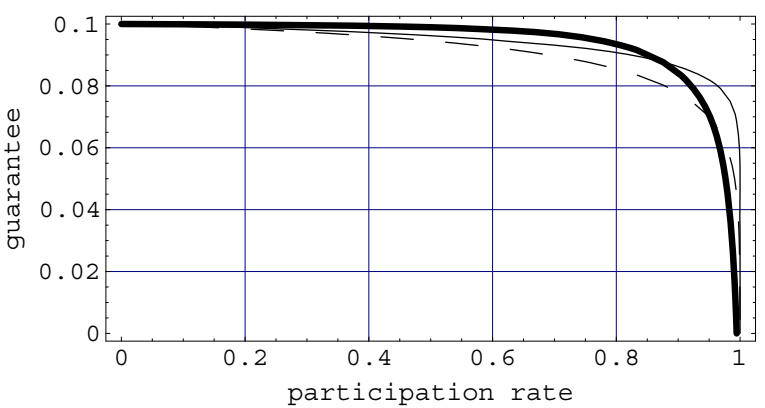

Figure 4: Long maturity ( $T=15, r=0.08, g=0, g=0.02$ (dashed line))

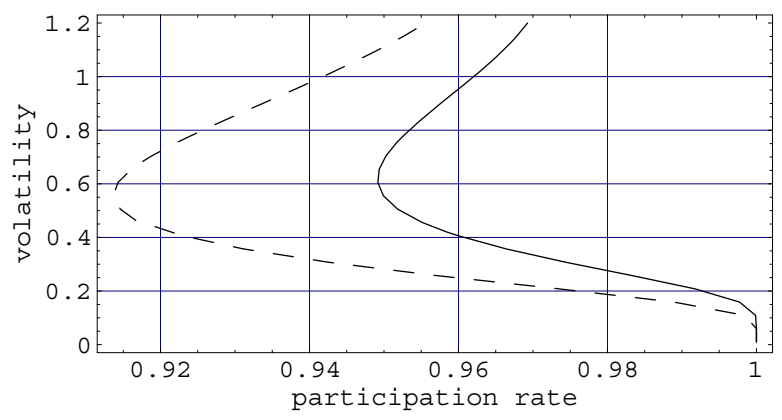

parameters $\left(\alpha^{*}, g_{i}^{*}\right)$ is restricted by the condition that at time $t_{i}$, the fair price of the $\mathrm{MRRG}^{(i)}$ must be given by $K_{i}$, i.e. we have

Corollary 2.4 If there is no upfront premium for the embedded option to pay, i.e. at $t_{i}$ the insured pays the amount $K_{i}$ to achieve the payoff $I^{(i)}\left(t_{N}\right)$ at $t_{N}$, then the fair zero-premium contract specification $\left(\alpha^{*}, g^{*}\right)$ has to satisfy the condition

(17) $1=\frac{B\left(t_{i}, t_{N}\right)}{G\left(t_{i}, t_{N}\right)}\left[1+\left(\frac{G\left(t_{i}, t_{N}\right)}{B\left(t_{i}, t_{N}\right)}\right)^{\alpha} e^{-\frac{1}{2} \alpha(1-\alpha) v_{t_{N}}^{2}\left(t_{i}\right)}\right.$

$$
\begin{aligned}
& \times \mathcal{N}\left(h_{1}^{(i)}\left(t_{i}, \frac{G\left(t_{i}, t_{N}\right)}{B\left(t_{i}, t_{N}\right)}\right)-(1-\alpha) v_{t_{N}}\left(t_{i}\right)\right) \\
& \left.-\mathcal{N}\left(h_{2}^{(i)}\left(t_{i}, \frac{1}{\frac{B\left(t_{i}, t_{N}\right)}{G\left(t_{i}, t_{N}\right)}}\right)\right)\right]
\end{aligned}
$$

Proof. The above corollary is a direct consequence of the fair pricing condition

$$
\operatorname{MRRG}^{(i)}\left(t_{i}, t_{N}\right)=K_{i}
$$

where $\operatorname{MRRG}^{(i)}\left(t_{i}, t_{N}\right)$ is given as in proposition 2.3 , i.e. equation (16) with $t=t_{i}$.

Note that for $\alpha^{*}=0$, (17) implies that $g^{*}$ must equal the risk-free rate, or more accurately (to 
cover the case of stochastic interest rates) the continuously compounded yield implied by the zerocoupon bond price $B\left(t_{i}, t_{N}\right)$ (or an appropriately weighted average thereof when one contract covers multiple investment dates $\left.t_{i}, 0 \leq i<N\right)$. This is also the maximum return than can be guaranteed without any additional upfront premium. For $\alpha^{*}=1$ (the maximum participation rate without any additional upfront premium), no return can be guaranteed, i.e. $g^{*}=-\infty$, and for $0<\alpha^{*}<1$, we have $g^{*}$ less than the risk-free rate.

The key parameter for the price of the option embedded in the minimum rate of return guarantee is the volatility $\sigma\left(t, t_{N}\right)$ of the forward price process of the benchmark index. Setting this to be constant in $t$, Figures 1-4 show the effect of this parameter on the fair combinations of participation rate and guaranteed return $\left(\alpha^{*}, g^{*}\right)$. Figures 1 and 2 plot the fair combinations $\left(\alpha^{*}, g^{*}\right)$ for three different volatility levels, respectively. Note that Figure 1 is in line with the initial intuition and the claim in Miltersen and Persson (1998), that the fair level of the guaranteed return is lower for higher volatilities of the benchmark index. However, this is not true in general. Due to the fact that the embedded option has a payoff that is concave above the exercise price, there exist volatility levels at which an increase in volatility leads to an increase in the fair guaranteed return, as illustrated in Figure 2. Thus there are potentially two very different volatility scenarios leading to the same fair combination $\left(\alpha^{*}, g^{*}\right)$, as Figure 4 shows. In other words, while for a given volatility of the benchmark portfolio there is a uniquely defined participation rate $\alpha$ which satisfies the fair pricing principle, the converse is not true. The absence of such a monotonicity of the value of the embedded option with respect to volatility becomes particularly relevant when the assumption of known, deterministic volatility is lifted and the more realistic case of uncertain volatility is considered, as will be done from the next section onwards.

\section{Hedging and Model Uncertainty}

Due to the typically very long maturity of the minimum-rate-of-return guarantees, there is a high degree of model risk associated with the fair valuation of these contracts. Pricing derivative financial instruments by arbitrage crucially relies on a set of assumptions on the evolution of the underly- ing assets. In particular, the price of the MRRG which is given in Proposition 2.3 is only valid for a deterministic volatility of the forward price of the benchmark index. Under this model assumption, the payoff of the MRRG can be replicated by continuously rebalancing a portfolio consisting of a position $\varphi^{X}$ in the index $X$ and a position $\varphi^{B}$ in the zero-coupon bond maturing at $t_{N}$. This hedging strategy $\varphi^{\mathrm{MRRG}}=\left(\varphi^{X}, \varphi^{B}\right)$ is given by

Proposition 3.1 In a model where the forward index process $X$ is a lognormal martingale under the $t_{N}$ forward measure $\mathbf{P}_{t_{N}}$, as in equation (6), it holds that the (perfect) hedging strategy $\varphi=$ $\left(\varphi_{t}\right)_{t_{i} \leq t \leq t_{N}}$ for the $M R R G^{(i)}$ in terms of the assets $X$ and $B$, i.e. $\varphi=\left(\varphi^{X}, \varphi^{B}\right)$, is given by

$$
\begin{aligned}
& \varphi_{t}^{X}=\alpha \frac{K_{i} B\left(t, t_{N}\right)}{X_{t} G\left(t_{i}, t_{N}\right)}\left(\frac{\frac{X_{t}}{X_{t_{i}}}}{\frac{B\left(t, t_{N}\right)}{G\left(t_{i}, t_{N}\right)}}\right)^{\alpha} e^{-\frac{1}{2} \alpha(1-\alpha) v_{t_{N}}^{2}(t)} \\
& \times \mathcal{N}\left(h_{1}^{(i)}\left(t, \frac{\frac{X_{t}}{X_{t_{i}}}}{\frac{B\left(t, t_{N}\right)}{G\left(t_{i}, t_{N}\right)}}\right)-(1-\alpha) v_{t_{N}}(t)\right), \\
& \text { (19) } \varphi_{t}^{B}=\frac{M R R G^{(i)}\left(t, t_{N}\right)-\varphi_{t}^{X} X_{t}}{B\left(t, t_{N}\right)} \text {. }
\end{aligned}
$$

Proof. The delta of the MRRG ${ }^{(i)}$ is obtained by taking the partial derivative of equation (16) with respect to $X$, i.e. in terms of the embedded power option it holds

$$
\varphi_{t}^{X}=N(\alpha, g) \Delta^{P O}\left(t, X_{t} ; K(\alpha, g)\right)
$$

By standard theory, i.e. Black and Scholes (1973), Harrison and Pliska (1981) and onwards, this leads to a self-financing portfolio strategy, which replicates the payoff of the MRRG at maturity.

Thus the fairness of the price of the MRRG is justified by two arguments: Firstly, the portfolio value $V$ where

$$
V_{t}(\varphi):=\varphi_{t}^{X} X_{t}+\varphi_{t}^{B} B\left(t, t_{N}\right)
$$

is equal to the MRRG's payoff at maturity $t_{N}$, i.e. $V_{t_{N}}(\varphi)=I^{(i)}\left(t_{N}\right)$. Secondly, the incremental change of the portfolio value $V$ is determined by the changes in the underlying asset prices, i.e.

$$
d V_{t}(\varphi)=\varphi_{t}^{X} d X_{t}+\varphi_{t}^{B} d B\left(t, t_{N}\right)
$$

as the strategy is self-financing in the sense that there are no further inflows or outflows of funds 
after the initial investment $V_{0}(\varphi)$. Thus, to prevent arbitrage opportunities, the fair price must be

$$
\operatorname{MRRG}^{(i)}\left(t, t_{N}\right)=V_{t}(\varphi)
$$

or may alternatively, as in Section 2, be calculated using the martingale approach, i.e. by taking expectations under the appropriate equivalent martingale measure.$^{10} \mathrm{It}$ is worth mentioning that the self-financing property is invariant with respect to a change of numeraire, i.e. $\varphi$ is self-financing iff

(24) $\quad d V_{t}^{*}(\varphi)=\varphi_{t}^{X} d X_{t}^{*}$

where $V_{t}^{*}(\varphi)=\varphi_{t}^{X} X_{t}^{*}+\varphi_{t}^{B}$ and $X_{t}^{*}=\frac{X_{t}}{B\left(t, t_{N}\right)}$.

The self-financing property of the hedging strategy crucially relies on the correctness of the model assumptions used to derive the hedge, i.e. that the forward price of the index evolves according to Equation (6) with a known, deterministic volatility $\sigma\left(t, t_{N}\right)$. This is the Achilles' Heel of Black/Scholestype pricing. The deterministic volatility assumption has been repeatedly empirically invalidated and in fact the "implied volatility smile" present in traded option prices directly contradicts it. On the other hand, none of the many models extending Black/Scholes to supposedly more realistic volatility assumptions have gained dominant acceptance in academia or in practice. Thus for any type of volatility assumption we are faced with a considerable amount of risk that the model may be wrong - this is what is commonly termed "model risk". Given the very long maturities involved, conservative hedging (and therefore pricing) of the MRRG should be driven by a strategy which is robust with respect to model misspecification.

To this end, suppose that the true dynamics of the index process do not coincide with the assumed dynamics as given by Equation (6). To highlight the difference of assumed and true volatility structure, we use the convention that the assumed volatility is denoted with a tilde, i.e. the volatility $\sigma\left(t, t_{N}\right)$ of Equation (6) is written in the following as $\tilde{\sigma}\left(t, t_{N}\right)$. The true process is specified to be as general as possible while tractable, i.e. we assume that the true index process is given by a diffusion process not necessarily identical to the setting which is described by Equation (6). A diffusion process set-

\footnotetext{
10 The equivalence of the two approaches follows from Ito's lemma and the Feynman/Kac theorem. For further reading on this topic, see for example the standard text of Musiela and Rutkowski (1997).
}

ting is understood in the sense that the martingale part $d X_{t}^{*}$ of the Doob-Meyer decomposition of the process $X^{*}$ can be written in the form

$$
d\left(X_{t}^{*}\right)^{M}=X_{t}^{*} \sigma_{t}\left(t_{N}\right) d W(t)
$$

where $\sigma$ is not necessarily equal to the $\tilde{\sigma}$ of Equation (6) and might, in particular, be stochastic. If the true dynamics (25) are not identical to the dynamics (6) assumed in constructing the hedge, the hedging strategy will no longer be self-financing. Defining the in- and outflows of funds for the strategy $\varphi$ in terms of money paid at $t_{N}$, we have the "forward cost process"

$$
L_{t}^{*}(\varphi)=V_{t}^{*}(\varphi)-V_{0}^{*}(\varphi)-\int_{0}^{t} \varphi_{u}^{X} d X_{u}^{*}
$$

The self-financing property is equivalent to the requirement that $L_{t}^{*}=0$ for all $0 \leq t \leq t_{N}$.

The results below are based on a well-known representation of the cost process $L^{*}$, which is summarised in the following proposition.

Proposition 3.2 Consider a European contingent claim $C$ with underlying $X$. If the true forward index dynamics $X^{*}$ are given by Equation (25), while the hedging strategy is calculated on the basis of Equation (6), then the costs of hedging are given by

$$
\begin{aligned}
L_{t}^{*}(\varphi)= & \frac{1}{2} \int_{0}^{t}\left(X_{u}^{*}\right)^{2} \tilde{C}_{x x}^{*}\left(u, X_{u}^{*}\right) \\
& \times\left(\left(\sigma_{u}^{t_{N}}\right)^{2}-\left(\tilde{\sigma}^{2}\left(u, t_{N}\right)\right)\right) d u,
\end{aligned}
$$

where $\tilde{C}^{*}$ denotes the assumed forward price of the claim $C, C_{x x}^{*}$ denotes the second partial derivative with respect to $X^{*}$, i.e. the assumed (forward) gamma.

\section{Proof. See Appendix E.}

It is worth mentioning that in case of a standard option $L^{*} \leq 0$ (respectively $L^{*} \geq 0$ ) holds for $\tilde{\sigma} \geq \sigma$ $(\tilde{\sigma} \leq \sigma)$. This is in fact true for all convex payoff profiles, since in these cases the (assumed) gamma does not change its sign. Similarly, the converse holds for concave payoff profiles. Thus, if the assumed volatility dominates the true volatility, the assumed price is an upper (lower) bound for the fair price of the convex (concave) payoff, and the converse also holds. However, an application of 
proposition 3.2 to the payoff of the $\mathrm{MRRG}^{(i)}$ results in

$$
\begin{aligned}
& L_{t}^{*}\left(\varphi_{M R R G^{(i)}}\right)=\frac{1}{2} N^{(i)}\left(\alpha, g_{i}\right) \\
& \quad \times \int_{0}^{t}\left(X_{u}^{*}\right)^{2} \frac{\gamma_{u}^{P O}\left(K=K^{(i)}\left(\alpha, g_{i}\right)\right)}{B\left(u, t_{N}\right)} \\
& \quad \times\left(\sigma_{u}^{2}\left(T_{N}\right)-\tilde{\sigma}^{2}\left(u, t_{N}\right)\right) d u
\end{aligned}
$$

where $N^{(i)}\left(\alpha, g_{i}\right)$ and $\left.K^{(i)}\left(\alpha, g_{i}\right)\right)$ are given in Lemma 2.1; $\gamma^{P O}$ is given in Theorem 2.2. Inserting $N^{(i)}, K^{(i)}$ and $\gamma^{P O}$ yields:

Proposition 3.3 Assume that the hedger uses the misspecified volatility $\tilde{\sigma}\left(t, t_{N}\right)$ instead of the true volatility $\sigma_{t}^{t_{N}}$. The discounted cost process $L^{*}$ of the $M R R G^{(i)}$ is given by

$$
\begin{aligned}
& d L_{t}^{*}\left(\varphi_{M R R G^{(i)}}\right)=\alpha \frac{K_{i}}{G\left(t_{i}, t_{N}\right)} e^{-\frac{1}{2} \alpha(1-\alpha) \tilde{v}_{t_{N}}^{2}(t)} \frac{Z_{t}^{\alpha}}{2} \\
& \quad \times\left(\frac{\mathcal{N}^{\prime}\left(h_{1}^{(i)}\left(t, Z_{t}\right)-(1-\alpha) \tilde{v}_{t_{N}}(t)\right)}{\tilde{v}_{t_{N}}(t)}\right. \\
& \left.\quad-(1-\alpha) \mathcal{N}\left(h_{1}^{(i)}\left(t, Z_{t}\right)-(1-\alpha) \tilde{v}_{t_{N}}(t)\right)\right) \\
& \quad \times\left(\left(\sigma_{t}^{t_{N}}\right)^{2}-\tilde{\sigma}^{2}\left(t, t_{N}\right)\right) d t,
\end{aligned}
$$

where $Z_{t}:=\frac{X_{t} / X_{t_{i}}}{B\left(t, t_{N}\right) / G\left(t_{i}, t_{N}\right)}$.

Note that the gamma of the MRRG can indeed change sign. This is due to the fact that the payoff of the MRRG is convex over some regions of $X_{T}$ and concave over others. Thus, neither does an overestimation of true volatility yield an upper arbitragefree price bound, nor does an underestimation of the true volatility yield a lower arbitrage-free price bound.

A conservative insurance company should take into account the high degree of model risk due to the fact that future volatility is uncertain. Therefore the contract parameters $\left(\alpha^{*}, g_{i}^{*}\right)$ need to be chosen such that the premium $K_{i}$ is given by the (lowest) upper price bound of the MRRG ${ }^{(i)}$. In particular, inserting an upper volatility bound in Equation (17) does not yield a conservative determination of contract parameters $\left(\alpha^{*}, g_{i}^{*}\right)$. Along the lines of the "uncertain volatility model" of Avellaneda, Levy and Parás (1995), assume that the true asset price dynamics are given by Equation 25 , with $\sigma_{t} \in\left[\sigma_{\min }, \sigma_{\max }\right]$ a.s. for all $t \in\left[t_{i}, t_{N}\right]$. It is well known that the lowest upper price bound for mixed payoff profiles is then the solution of the Black/Scholes/Barenblatt (BSB) equation ${ }^{11}$, as shown in Avellaneda, Levy and Parás (1995). Thus in case of the MRRG ${ }^{(i)}$ one would need to determine $\left(\alpha^{*}, g_{i}^{*}\right)$ by solving a modified version of (17), where the right-hand side is given in terms of the solution to a BSB equation. This is not tractable. The easiest alternative to incorporate the model risk might be given by an additional up-front risk premium $\Pi^{(i)}$ such that

(30) $\Pi^{(i)}=$ Lowest upper price bound $-\mathrm{MRRG}^{(i)}$

where the price of the MRRG ${ }^{(i)}$ is calculated according to a deterministic volatility structure and $\left(\alpha^{*}, g_{i}^{*}\right)$ are determined according to Equation (17). However, besides raising an unwanted additional up-front premium, this is still not easy to implement. Thus, the aim of the following sections is firstly to establish a (meaningful) price bound, which allows us to determine $\left(\alpha^{*}, g_{i}^{*}\right)$ conservatively, i.e. such that the conservative value of the insurance contract is given by the premium $K_{i}$. Secondly, the goal is to find a tractable and robust hedging strategy under uncertain volatility.

\section{Static Hedging}

\subsection{Hedging with standard options}

The most straightforward and robust solution to the hedging problem is possible if standard (call or put) options on $X$ with maturity $t_{N}$ are traded in the market. By Lemma 2.1, we have a positive payoff of the embedded option if $X_{t_{N}}>X_{t_{i}} / G\left(t_{i}, t_{N}\right)$. Writing this positive payoff as a function of the terminal index value,

(31) $f(x)=\frac{K_{i}}{G\left(t_{i}, t_{N}\right)}\left(\left(\frac{G\left(t_{i}, t_{N}\right)}{X_{t_{i}}} x\right)^{\alpha}-1\right)$

This yields the lowermost curve in Figure 5. We see that we can dominate this payoff by the payoff of a number of call options with exercise price $\hat{K}_{0}=$ $X_{t_{i}} / G\left(t_{i}, t_{N}\right)$ (the uppermost curve in Figure 5 ). The number of call options required is given by the

${ }^{11}$ In general, a Black-Scholes-Barenblatt equation is a Hamilton-Jacobi-Bellman equation and is linked to a stochastic control problem. The link between a smooth solution of a BSB equation and a superhedging strategy is discussed in Vargiolu (2001). Details on the numerical solution are discussed in Pooley, Forsyth and Vetzal (2003). In case of convex payoff profiles the solution is of course simply given by the Black/Scholes price composed at the upper volatility bound. 
Table 1: Optimal adjustment of the static superhedge

\begin{tabular}{|c|c|c|c|c|c|c|c|c|c|}
\hline \multirow[b]{2}{*}{$m$} & \multirow{2}{*}{\multicolumn{2}{|c|}{ Call option short positions }} & \multirow{2}{*}{\multicolumn{3}{|c|}{ Call option strikes }} & & & \multicolumn{2}{|c|}{ Overpricing } \\
\hline & & & & & & & & absolute & relative (\%) \\
\hline 1 & 2.37 & & 465.4 & & & & & 20.7358 & 5.26998 \\
\hline 2 & 1.66 & & 322.3 & 1201.1 & & & & 8.9823 & 2.282844 \\
\hline 3 & 1.08 & 1.10 & 271.4 & 697.8 & 2014.0 & & & 5.0214 & 1.27617 \\
\hline 4 & 0.89 & 0.92 & 246.3 & 524.5 & 1138.2 & 2890.8 & & 3.2089 & 0.81553 \\
\hline 5 & 0.89 & 0.710 .81 & 229.4 & 428.8 & 801.8 & 1584.0 & 3700.0 & 2.2298 & 0.56669 \\
\hline
\end{tabular}

Figure 5: Dominating the power option payoff with a long position in a call option and with a long/short position in two call options (parameter constellation as for Table 1)

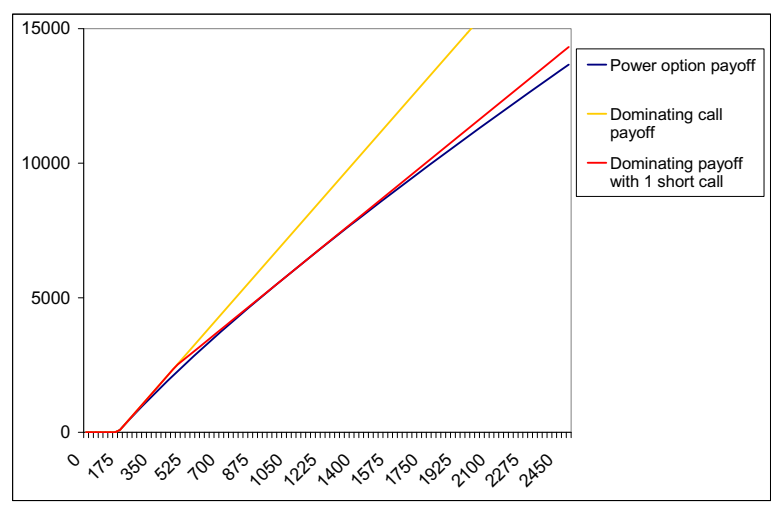

slope of the positive payoff in $\hat{K}_{0}$, i.e.

$$
\left.\frac{\partial I^{(i)}\left(t_{N}\right)}{\partial X_{t_{N}}}\right|_{X_{t_{N}} \backslash \hat{K}_{0}}=\alpha \frac{K_{i}}{X_{t_{i}}}
$$

Thus by combining a short position in the minimum-rate-of-return guarantee with a long position of $\alpha K_{i} / X_{t_{i}}$ call options with strike $\hat{K}_{0}$, one could do away with delta-hedging altogether: The payoff of the call options dominates the payoff of the MRRG, so the position does not have any downside risk. However, this is typically not practicable, as the call option will be quite expensive compared to the fair price of the MRRG.

On the other hand, any piecewise linear payoff can be replicated by a portfolio of standard options, and the nonlinear payoff of the MRRG can be approximated by a piecewise linear function. The accuracy of the approximation is only limited by the number different strikes in the portfolio of standard options. Suppose that we have hedged a short position in the minimum rate of return guarantee with a long position of $\alpha K_{i} / X_{t_{i}}$ call options with strike $\hat{K}_{0}$. We can now proceed to reduce the cost of the hedge by going short call options with higher strikes - the payoff obtained by taking a short position in call options of one additional strike is given by the middle curve in Figure 5 . The procedure is as follows. Let $x_{0}=\hat{K}_{0}$. For a given set of points $\left\{x_{1}, \ldots, x_{m}\right\}>\hat{K}_{0}$, we can construct a portfolio of short positions in call options maturing in $t_{N}$, such that the combined payoff of this portfolio and the long position of $\alpha K_{i} / X_{t_{i}}$ call options with strike $\hat{K}_{0}$ dominates the payoff of the MRRG and is tangent to $f(x)$ in $\left\{x_{0}, x_{1}, \ldots, x_{m}\right\}$. The corresponding strikes of the sold call options are given by

$$
\begin{aligned}
& x_{j}^{*}=\frac{f\left(x_{j-1}\right)-f\left(x_{j}\right)+f^{\prime}\left(x_{j}\right) x_{j}-f^{\prime}\left(x_{j-1}\right) x_{j-1}}{f^{\prime}\left(x_{j}\right)-f^{\prime}\left(x_{j-1}\right)}, \\
& j>0
\end{aligned}
$$

and number of options sold at each strike is

$$
n_{j}=f^{\prime}\left(x_{j-1}\right)-f^{\prime}\left(x_{j}\right), \quad j>0
$$

For a given number of strikes, one can minimise the total cost of hedging the MRRG by solving

$$
\begin{aligned}
& \max _{\left\{x_{1}, \ldots, x_{m}\right\}>\hat{K}_{0}} \sum_{j=1}^{m} n_{i}\left[X_{t_{i}}\right. \\
& \times \mathcal{N}\left(\frac{\ln \frac{X_{t_{i}}}{x_{j}^{*} B\left(t_{i}, t_{N}\right)}+\frac{1}{2} \tilde{v}_{t_{N}}^{2}\left(t_{i}\right)}{v_{t_{N}}\left(t_{i}\right)}\right) \\
& \left.-x_{j}^{*} B\left(t_{i}, t_{N}\right) \mathcal{N}\left(\frac{\ln \frac{x_{t_{i}}}{x_{j}^{*} B\left(t_{i}, t_{N}\right)}-\frac{1}{2} \tilde{v}_{t_{N}}^{2}\left(t_{i}\right)}{v_{t_{N}}\left(t_{i}\right)}\right)\right]
\end{aligned}
$$

Obviously, as $m \rightarrow \infty$, the cost of statically replicating the MRRG approaches its fair value, but already for small values of $m$ the approximation is quite good, as the following example illustrates for the Black/Scholes case of known and constant volatility.

Consider a minimum-rate-of-return guarantee of $g=5 \%$ on an initial investment of $K_{i}=1000$ at time $t_{i}=0$ with maturity $t_{N}=10$ years. Let the 
Figure 6: Embedded option payoff approximated from above by portfolios of standard options

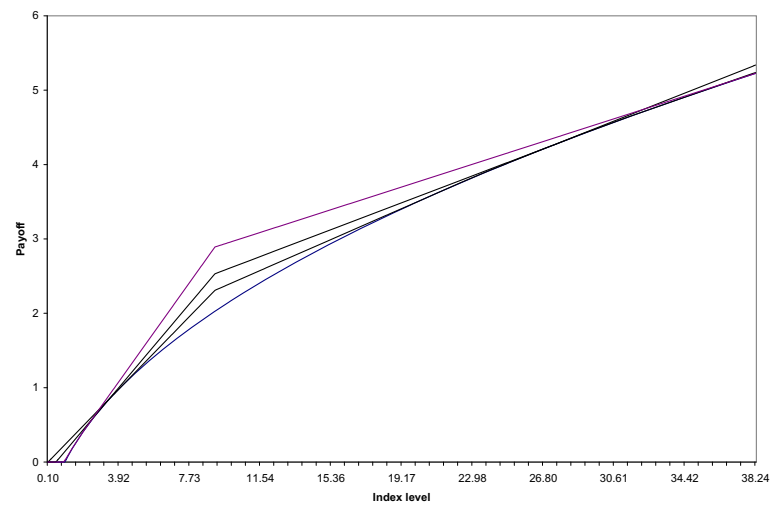

risk-free rate for this time horizon be $10 \%$, the current level of the benchmark index $X_{t_{i}}=100$, and the volatility of the index $40 \%$ per annum. The "fair price" participation rate is the $\alpha=0.819768$. The value of the power option component of the MRRG is then given by

$$
K_{i}-K_{i} e^{(g-r)\left(t_{N}-t_{i}\right)}=393.469
$$

$\alpha K_{i} / X_{t_{i}}=8.19768$ call options with strike $\hat{K}_{0}=$ 164.872 are worth 493.135 , i.e. the superhedge is too expensive by 99.6653 or $25.33 \%$. Table 1 shows the optimal strikes and short positions in $m$ additional call options (for $m=1, \ldots, 5$ ) and the remaining absolute and relative overpricing.

\subsection{Bounds on contract parameters implied by market prices}

The static superhedge and an analogously constructed subhedge can be used to infer lower and upper bounds on arbitrage-free zero-premium combinations of participation rate and guaranteed return. Since for exchange-traded options the exercise prices are fixed by the contract specifications, the construction of the hedge differs slightly from the previous section.

To set up a superhedge, consider Figure 6. For a given strike $\bar{x}_{0}^{*} \leq \hat{K}_{0}$, determine the line tangent to $f(x)$, which goes through the point $\left(\bar{x}_{0}^{*}, 0\right)$. This is the payoff of $\bar{N}_{0}$ call options with strike $\bar{x}_{0}^{*}$, where $\bar{N}_{0}=f^{\prime}\left(\bar{x}_{0}\right)$ and $\bar{x}_{0}$ solves

(37) $f\left(\bar{x}_{0}\right)=\left(\bar{x}_{0}-\bar{x}_{0}^{*}\right) f^{\prime}\left(\bar{x}_{0}\right)$

Subsequently, from the set of strikes fixed by the contract specifications, choose the next strike $\bar{x}_{i}^{*}$ to be the smallest available strike greater than $\bar{x}_{i-1}$
Figure 7: Embedded option payoff approximated from below by a portfolio of standard options

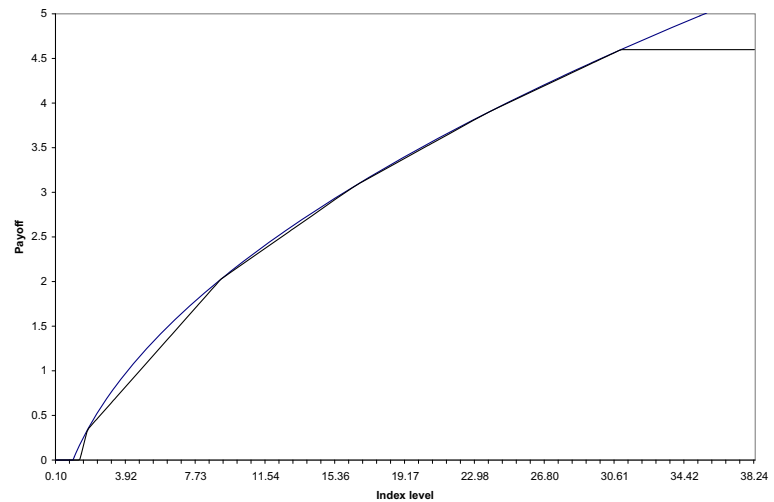

and go short $\bar{N}_{i}=f^{\prime}\left(\bar{x}_{i-1}\right)-f^{\prime}\left(\bar{x}_{i}\right)$ call options at this strike, where $\bar{x}_{i}$ solves

$$
\begin{aligned}
f\left(\bar{x}_{i}\right)= & f\left(\bar{x}_{i-1}\right)+f^{\prime}\left(\bar{x}_{i-1}\right)\left(\bar{x}_{i}^{*}-\bar{x}_{i-1}\right) \\
& +f^{\prime}\left(\bar{x}_{i}\right)\left(\bar{x}_{i}-\bar{x}_{i}^{*}\right)
\end{aligned}
$$

Repeat this process until no further strikes are available.

Figure 6 graphs the superhedge payoffs for three different starting strikes $\bar{x}_{0}^{*}$. Clearly, the cheapest superhedge is not necessarily given by starting with the highest available strike less than or equal to $\hat{K}_{0}$. Rather, this will depend on the position of the other available strikes as well as the market prices of the respective options.

For the construction of the subhedge, consider Figure 7. Here, a long position is taken in the call option with the smallest available strike $\underline{x}_{0} \geq \hat{K}_{0}$. The number of options bought is given by

(39) $\underline{N}_{0}=\frac{f\left(\underline{x}_{1}\right)-f\left(\underline{x}_{0}\right)}{\underline{x}_{1}-\underline{x}_{0}}$

where $\underline{x}_{1}$ denotes the next-greatest available strike. Short positions are then taken in call options for all available strikes $\underline{x}_{i}>\underline{x}_{0}, 1 \leq i \leq m$, where the number of options sold is given by

(40) $\quad \underline{N}_{i}=\frac{f\left(\underline{x}_{i}\right)-f\left(\underline{x}_{i-1}\right)}{\underline{x}_{i}-\underline{x}_{i-1}}-\frac{f\left(\underline{x}_{i+1}\right)-f\left(\underline{x}_{i}\right)}{\underline{x}_{i+1}-\underline{x}_{i}}$

for $1 \leq i<m$ and

$$
\underline{N}_{m}=\frac{f\left(\underline{x}_{m}\right)-f\left(\underline{x}_{m-1}\right)}{\underline{x}_{m}-\underline{x}_{m-1}}
$$

Applying this to market data, we use settlement prices for options on Sydney Futures Exchange SPI 200 stock index futures to calculate the bounds 
Figure 8: Bounds on fair $(g, \alpha)$ combinations implied by index option prices on the Sydney Futures Exchange on 30 March 2001

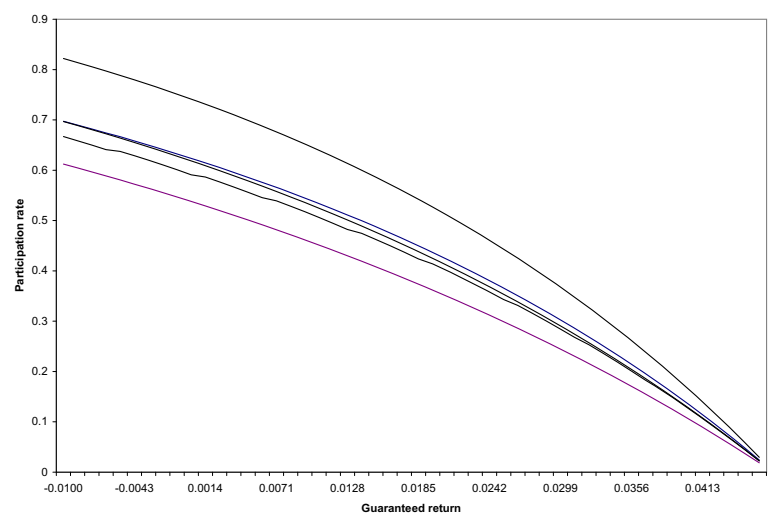

on arbitrage-free combinations of participation rate and guaranteed return $(\alpha, g)$. Table 2 in Appendix $\mathrm{G}$ summarises the relevant market data for 30 March 2001. The MRRG under consideration is on a single premium payment made on 30 March 2001 and the contract matures at the end of June 2002, the longest maturity for which market data is available. For the purpose of this indicative calculation, the distinction between futures and forwards was ignored. The discount factor for the June 2002 maturity was inferred from 90-day bank accepted bills futures also traded on the SFE and option prices were calculated using the forward version of the Black/Scholes formula (for the call options) and of (7) (for the embedded power option), i.e. substituting the June 2002 futures price for $X_{t} / B\left(t, t_{N}\right)$, thus making an explicit reference to the dividend yield unnecessary.

Figure 8 plots the resulting bounds on the fair $(g, \alpha)$ combinations. The outer bounds were calculated using (17), inserting the maximum (lower bound) and minimum (upper bound) implied volatility from Table 2 for $v_{t_{N}} .{ }^{12}$ The inner bounds are those given by the sub- and superhedge. The inner upper bound thus plots those combinations $(g, \alpha)$, for which the right-hand side of (84) equals $K_{i}$, with the value $P O\left(t, X_{t} ; K(\alpha, g)\right)$ substituted by the market value of the subhedge portfolio. Since the superhedge portfolio depends on the choice of $\bar{x}_{0}$, the inner lower bound plots the $(g, \alpha)$ satisfying the above condition with $P O\left(t, X_{t} ; K(\alpha, g)\right)$ substituted

\footnotetext{
${ }^{12}$ As noted in Section 2, neither the fair return guarantee nor the fair participation rate are necessarily monotonic in the volatility parameter. However, monotonicity holds in the particular example considered here.
}

by the smallest market value of the possible superhedge portfolios. For reference, the middle curve plots the $(g, \alpha)$ satisfying (17) with $v_{t_{N}}$ equal to the implied volatility of the at-the-money futures option.

We see that the static hedge bounds are quite tight. They would be widened somewhat if one were to take into account the bid-ask spreads, but they are certainly much tighter than the outer bounds given by the highest and lowest implied volatilities for this maturity. Note that by constructing static hedges of traded options for the MRRG, one is effectively pricing it in a manner consistent with the volatility "smile" observed in the market, independent of the validity of any given model assumptions.

There remains, however, the problem that this type of hedging can only be implemented if options of the desired maturity are traded in the market. For the typically very long maturities of the MRRG this is generally not the case, so we will look at alternatives to static hedging in the next section.

\section{Conservative contract specification based on a robust dynamic hedge}

\subsection{Robust hedging}

As discussed in Section 3, one source of difficulty in applying a dynamic delta-hedge is the potential for model misspecification. For options with convex (concave) payoffs, this problem can be mitigated by calculating the delta-hedging strategy using a higher (lower) volatility, resulting in a superhedge. The payoff of a (long position in the) minimum-rate-of-return guarantee is neither convex nor concave, but can be made concave by combining it with a short position of an appropriate number of standard options with the appropriate strike. First, note that the cost process given in Proposition 3.3 can be written as

$$
\begin{aligned}
& d L_{t}^{*}=\alpha \frac{K_{i}}{G\left(t_{i}, t_{N}\right)} e^{-\frac{1}{2} \alpha(1-\alpha) \tilde{v}_{t_{N}}^{2}(t)} \frac{Z_{t}^{\alpha}}{2} \\
& \times\left(\frac{\mathcal{N}^{\prime}\left(h_{1}^{(i)}\left(t, Z_{t}\right)\right)}{\tilde{v}_{t_{N}}(t)} e^{\frac{1}{2} \alpha(1-\alpha) \tilde{v}_{t_{N}}^{2}(t)} Z_{t}^{1-\alpha}\right. \\
& \left.-(1-\alpha) \mathcal{N}\left(h_{1}^{(i)}\left(t, Z_{t}\right)-(1-\alpha) \tilde{v}_{t_{N}}(t)\right)\right) \\
& \times\left(\left(\sigma_{t}^{t_{N}}\right)^{2}-\tilde{\sigma}^{2}\left(t, t_{N}\right)\right) d t
\end{aligned}
$$




\section{Combined payoffs of MRRG and standard option}

Figure 9: Long MRRG (thin line) and combined position with short call (thick line)

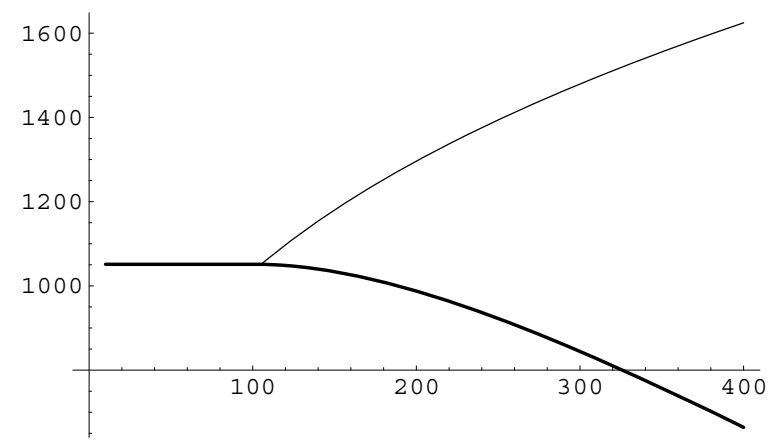

$$
\begin{aligned}
= & \left(\frac{1}{2} \alpha \frac{K_{i}}{X_{t_{i}}}\left(\frac{X_{t}}{B\left(t, t_{N}\right)}\right)^{2} \tilde{\gamma}_{t}^{*}\left(\hat{K}=\frac{X_{t_{i}}}{G\left(t_{i}, t_{N}\right)}\right)\right. \\
& -\alpha \frac{K_{i}}{G\left(t_{i}, t_{N}\right)} e^{-\frac{1}{2} \alpha(1-\alpha) \tilde{v}_{t_{N}}^{2}(t)} \frac{Z_{t}^{\alpha}}{2}(1-\alpha) \\
& \left.\times \mathcal{N}\left(h_{1}^{(i)}\left(t, Z_{t}\right)-(1-\alpha) \tilde{v}_{t_{N}}(t)\right)\right) \\
& \times\left(\left(\sigma_{t}^{t_{N}}\right)^{2}-\tilde{\sigma}^{2}\left(t, t_{N}\right)\right) d t
\end{aligned}
$$

where

$$
\tilde{\gamma}_{t}^{*}\left(\hat{K}_{0}=\frac{X_{t_{i}}}{G\left(t_{i}, t_{N}\right)}\right)
$$

denotes the gamma of a standard (call or put) option with strike $\hat{K}_{0}$, maturing at $t_{N}$. The above cost process results from hedging a short position in the minimum rate of return guarantee, i.e. from creating a synthetic long position. Going long $\alpha K_{i} / X_{t_{i}}$ standard options at strike $\hat{K}_{0}$ and hedging this with a synthetic short position results in an additional cost process

$$
\begin{aligned}
& -\frac{1}{2} \alpha \frac{K_{i}}{X_{t_{i}}}\left(\frac{X_{t}}{B\left(t, t_{N}\right)}\right)^{2} \tilde{\gamma}_{t}^{*}\left(\hat{K}=\frac{X_{t_{i}}}{G\left(t_{i}, t_{N}\right)}\right) \\
& \quad \times\left(\left(\sigma_{t}^{t_{N}}\right)^{2}-\tilde{\sigma}^{2}\left(t, t_{N}\right)\right) d t
\end{aligned}
$$

so the net cost process becomes

$$
\text { (44) } \begin{aligned}
- & \alpha \frac{K_{i}}{G\left(t_{i}, t_{N}\right)} e^{-\frac{1}{2} \alpha(1-\alpha) \tilde{v}_{t_{N}}^{2}(t)} \frac{Z_{t}^{\alpha}}{2}(1-\alpha) \\
& \times \mathcal{N}\left(h_{1}^{(i)}\left(t, Z_{t}\right)-(1-\alpha) \tilde{v}_{t_{N}}(t)\right) \\
& \times\left(\left(\sigma_{t}^{t_{N}}\right)^{2}-\tilde{\sigma}^{2}\left(t, t_{N}\right)\right) d t
\end{aligned}
$$

and we achieve a superhedge if $\left(\sigma_{t}^{t_{N}}\right)^{2} \geq \tilde{\sigma}^{2}\left(t, t_{N}\right)$. Unsurprisingly, the strike of the standard option
Figure 10: Long MRRG (thin line) and combined position with short put (thick line)

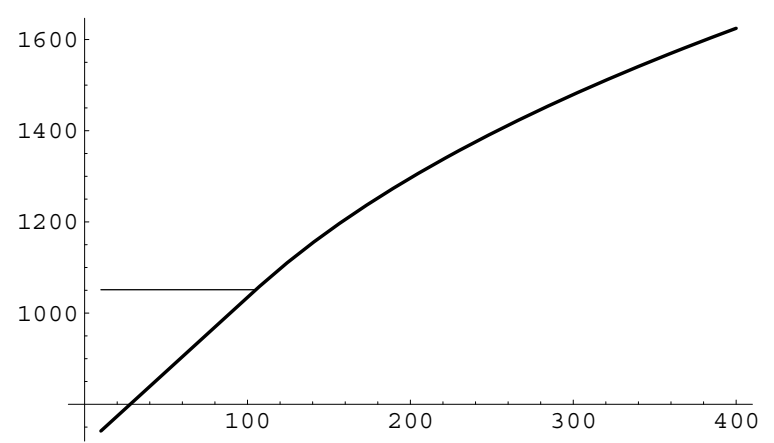

is the point above which the holder of the MRRG begins to participate in gains of $X_{t_{N}}$ and the number of standard options required corresponds to the initial slope of the participation, i.e.

(45) $\left.\frac{\partial I^{(i)}\left(t_{N}\right)}{\partial X_{t_{N}}}\right|_{X_{t_{N} \searrow \hat{K}_{0}}}=\alpha \frac{K_{i}}{X_{t_{i}}}$

The resulting payoff of a long position in the MRRG combined with a short position in the standard option is concave, as Figures 9 and 10 illustrate for the call and put, respectively.

Proposition 5.1 If the "true" model is given by Equation (25) with $\sigma_{t}\left(t_{N}\right) \in\left[\sigma_{\min }, \sigma_{\max }\right]$ for all $t_{i} \leq t \leq t_{N}$, it holds that

(a) A conservative upper price bound of the $M R R G^{(i)}$ is given by

$$
\begin{aligned}
& \alpha \frac{K_{i}}{X_{t_{i}}} \operatorname{Call}\left(t, t_{N} ; \hat{K}_{0}=\frac{X_{t_{i}}}{G\left(t_{i}, t_{N}\right)} ; \sigma_{\text {max }}\right) \\
& +\left[\operatorname{MRRG}^{(i)}\left(t, t_{N} ; \sigma_{\min }\right)\right. \\
& \left.-\alpha \frac{K_{i}}{X_{t_{i}}} \operatorname{Call}\left(t, t_{N} ; \hat{K}_{0}=\frac{X_{t_{i}}}{G\left(t_{i}, t_{N}\right)} ; \sigma_{\text {min }}\right)\right]
\end{aligned}
$$

where $\operatorname{MRRG}^{(i)}(\cdot)$ is given by (16) and Call( $\left.\cdot\right)$ denotes the Black/Scholes call option pricing formula.

(b) A the conservative choice of $\left(\alpha^{*}, g_{i}^{*}\right)$ is given such that

(47)

$$
\begin{aligned}
K_{i}= & \operatorname{MRRG}^{(i)}\left(t_{i}, t_{N} ; \sigma_{\min }\right) \\
& +\alpha \frac{K_{i}}{X_{t_{i}}}\left[\operatorname{Call}\left(t_{i}, t_{N} ; \hat{K}_{0} ; \sigma_{\max }\right)\right. \\
& \left.-\operatorname{Call}\left(t_{i}, t_{N} ; \hat{K}_{0} ; \sigma_{\min }\right)\right]
\end{aligned}
$$




\section{Conservative Contract Parameter Combinations}

(Guaranteed return $g$ versus participation rate $\alpha$ )

Figure 11: For $\sigma_{\min }=\sigma_{\max }=0.1$ (thin line),

$\sigma_{\min }=\sigma_{\max }=0.8($ dashed line $)$ and $\sigma_{\min }=0.1$ combined with $\sigma_{\max }=0.8$ (thick line)

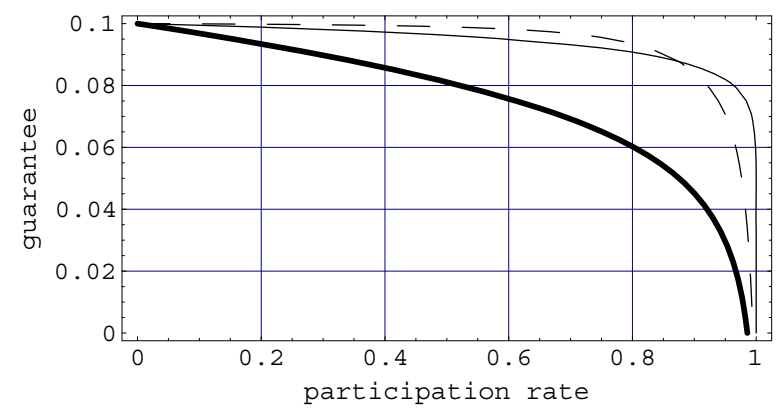

(c) A conservative robust hedging strategy in terms of the assets $X$ and $B$ is given by

$$
\begin{aligned}
\Delta_{t}^{X}= & \alpha \frac{K_{i}}{X_{t_{i}}}\left(\mathcal{N}\left(t, X_{t} ; v_{\max }(t)\right)\right. \\
& \left.-\mathcal{N}\left(t, X_{t} ; v_{\min }(t)\right)\right)+\varphi_{t}^{X}\left(v_{\min }(t)\right) \\
\Delta_{t}^{B}= & \frac{(46)-\Delta_{t}^{X} X_{t}}{B\left(t, t_{N}\right)}
\end{aligned}
$$

where $\varphi_{t}^{X}$ is given as in Proposition 3.1, $h_{1}(t, z)$ as in Theorem 2.2 and

$$
\begin{aligned}
& v_{\max }(t):=\sqrt{\int_{t}^{t_{N}} \sigma_{\max }^{2}\left(s, t_{N}\right) d s} \\
& v_{\min }(t):=\sqrt{\int_{t}^{t_{N}} \sigma_{\min }^{2}\left(s, t_{N}\right) d s}
\end{aligned}
$$

(d) The cost process for the above strategy is given by

$$
\text { (49) } \begin{aligned}
& d L_{t}^{*}=\left[\frac{1}{2} \alpha \frac{K_{i}}{X_{t_{i}}}\left(\frac{X_{t}}{B\left(t, t_{N}\right)}\right)^{2}\right. \\
\times & \operatorname{Call}_{x x}\left(t, t_{N} ; \hat{K}_{0} ; \sigma_{\max }\right)\left(\left(\sigma_{t}^{t_{N}}\right)^{2}-\sigma_{\max }^{2}\left(t, t_{N}\right)\right) \\
- & \alpha \frac{K_{i}}{G\left(t_{i}, t_{N}\right)} e^{-\frac{1}{2} \alpha(1-\alpha) \tilde{v}_{\text {min }}^{2}(t)} \frac{Z_{t}^{\alpha}}{2}(1-\alpha) \\
\times & \mathcal{N}\left(h_{1}^{(i)}\left(t, Z_{t}\right)-(1-\alpha) \tilde{v}_{\min }(t)\right) \\
\times & \left.\left(\left(\sigma_{t}^{t_{N}}\right)^{2}-\sigma_{\min }^{2}\left(t, t_{N}\right)\right)\right] d t
\end{aligned}
$$

Proof. See Appendix F.

Figures 11 and 12 illustrate the conservative contract parameter combinations resulting from
Figure 12: For $\sigma_{\min }=0.1$ while $\sigma_{\max }=0.4$ (thin line), $\sigma_{\max }=0.5$ (dashed line) and $\sigma_{\max }=0.8$ (thick line)

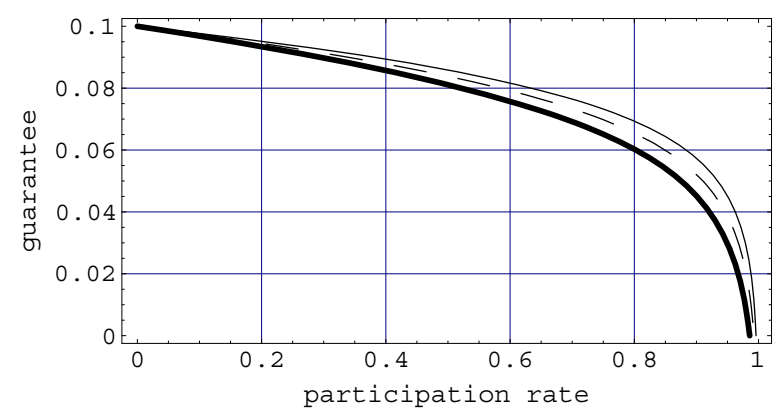

Proposition 5.1(b). The thick line of Figure 11 plots combinations of participation rates $\alpha$ and minimum-rate-of-return guarantees $g$, which satisfy Equation (47) and thus represent conservative contract specifications with respect to an uncertain volatility model in the sense of Equation (25), where $\sigma \in\left[\sigma_{\min }, \sigma_{\max }\right]$. The parameters $r$ and $t_{N}$ are chosen as in Figure 2, i.e. $r=0.1$ and $t_{N}=30$. Figure 11 also contrasts the conservative contract combinations to those resulting from the fair pricing condition of Equation (17), when volatility is set to the upper (dashed line) or lower (thin line) volatility bound. Note that the participation rate for a given guarantee is substantially reduced compared to the fair combinations calculated at either volatility bound and, as illustrated in Figure 12, the effect increases as the width of the uncertain volatility interval increases. On the one hand, this clearly demonstrates the importance of a careful choice of participation rate and guaranteed return on the part of the insurer. On the other hand, it means that although this approach to conservatively determining contract parameters is very tractable, it should only be seen as a first step toward calculating the "best" conservative parameter combination.

\subsection{Improving the price bounds}

In a sense, the robust dynamic hedge proposed above is closely related to the construction of a static superhedge in Section 4. Instead of buying a number of call options with strike $\hat{K}_{0}$ (since supposedly options with the desired maturity are not available in the market), a synthetic long position in these options is superreplicated by a dy- 
Figure 13: Straight line dominating $f(x)$

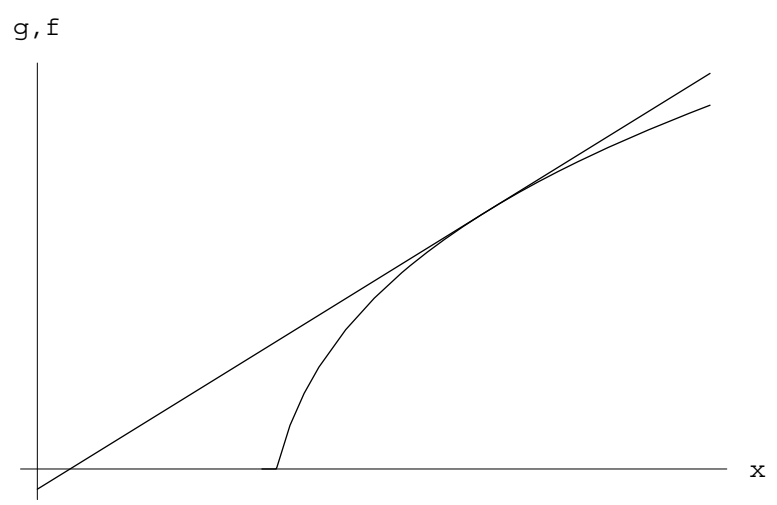

namic delta-hedging strategy at the upper volatility bound. Instead of reducing the total cost of the hedge by going short (market traded) call options, the initial investment in the hedge is reduced by a synthetic short position in the call option, superreplicated by a dynamic strategy at the lower volatility bound.

Taking this analogy to the static hedge further, the price bound resulting from a robust dynamic (Black/Scholes-type) hedge can be improved by optimising the choice of the dominating payoff. ${ }^{13}$ As before, denote by $f(x)$ the positive part of the payoff of the embedded power option, i.e.

$$
\begin{aligned}
f(x) & =\frac{K_{i}}{G\left(t_{i}, t_{N}\right)}\left(\left(\frac{x G\left(t_{i}, t_{N}\right)}{X_{t_{i}}}\right)^{\alpha}-1\right) \\
& =N^{(i)}\left(\alpha, g_{i}\right)\left(x^{\alpha}-K^{(i)}\left(\alpha, g_{i}\right)\right) \\
x & \geq \hat{K}_{0}=\frac{X_{t_{i}}}{G\left(t_{i}, t_{N}\right)}
\end{aligned}
$$

Thus the payoff can be written as $\max (0, f(x))$.

This payoff cannot be dominated by a concave function because of the $\max (0, \cdot)$ condition. Thus we must dominate $f(x)$ by a convex function, the tightest of which will be (by the separating hyperplane theorem) a straight line. When combined with the $\max (0, \cdot)$ requirement, this will be the payoff of a vanilla call with some strike less than or equal to $\hat{K}_{0}$, as one can see in Figure 13 .

As in Section 4, if the dominating payoff is tangent to $f(x)$ in $\bar{x}_{0}$, the strike of the corresponding

\footnotetext{
13 Branger and Mahayni (2006) call a hedging strategy which can be represented as the sum of Black/Scholes strategies a tractable hedge. They give a characterization of the optimal dominating payoff for arbitrary European claims. In particular, the cheapest tractable hedge for a power option is treated as an example.
}

standard call is given by

(51) $\bar{x}_{0}^{*}=\hat{K}\left(\bar{x}_{0}\right)=\bar{x}_{0}-\frac{f\left(\bar{x}_{0}\right)}{f^{\prime}\left(\bar{x}_{0}\right)}$

and the number of options required is $\bar{N}_{0}=f^{\prime}\left(\bar{x}_{0}\right)$. Writing the dominating payoff as

$$
g(x)=f^{\prime}\left(\bar{x}_{0}\right)\left[x-\hat{K}\left(\bar{x}_{0}\right)\right]^{+}
$$

we can represent the difference between the two payoffs as

$$
g(x)-f(x)=R(x)=R_{0}(x)-R_{1}(x)
$$

where

$$
\begin{aligned}
& R_{0}(x)=f^{\prime}\left(\bar{x}_{0}\right)\left[x-\hat{K}\left(\bar{x}_{0}\right)\right] 1_{\left\{\hat{K}\left(\bar{x}_{0}\right) \leq x<\hat{K}_{0}\right\}} \\
& \quad+\left(f^{\prime}\left(\bar{x}_{0}\right)\left[x-\hat{K}\left(\bar{x}_{0}\right)\right]-f(x)\right) 1_{\left\{\hat{K}_{0} \leq x<\bar{x}_{0}\right\}} \\
& R_{1}(x)=\left(f\left(\bar{x}_{0}\right)+f^{\prime}\left(\bar{x}_{0}\right)\left[x-\bar{x}_{0}\right]-f(x)\right) 1_{\left\{x \geq \bar{x}_{0}\right\}} \\
& \quad=\left[x-\bar{x}_{0}\right]^{+} f^{\prime}\left(\bar{x}_{0}\right)-\left[f(x)-f\left(\bar{x}_{0}\right)\right]^{+}
\end{aligned}
$$

The initial investment in the superhedge can be reduced by going short the residual $R_{1}(x)$. Note that $R_{1}(x)$ is convex $\left(R_{0}(x)\right.$ is a mixed payoff), so we can superreplicate the short position in $R_{1}(x)$ by hedging it at the lower volatility bound. Let $P\left(\bar{x}_{0}, R_{1}(x), \sigma_{\min }\right)$ denote the value of the payoff $R_{1}(x)$ for a given choice of $\bar{x}_{0}$, calculated using the lower volatility bound. The problem of finding the cheapest possible superhedge then becomes: Solve, for a given $X_{t}$,

$$
\begin{aligned}
\hat{\bar{x}}_{0}= & \arg \min _{\bar{x}_{0} \geq \hat{K}_{0}} f^{\prime}\left(\bar{x}_{0}\right) \operatorname{Call}\left(X_{t}, \hat{K}\left(\bar{x}_{0}\right), \sigma_{\max }, t_{N}\right) \\
& -P\left(\bar{x}_{0}, R_{1}(x), \sigma_{\min }\right)
\end{aligned}
$$

If the optimal $\hat{K}\left(\hat{\bar{x}}_{0}\right)$ lies below $\hat{K}_{0}$, there is also a residual for the interval $\left[\hat{K}\left(\hat{\bar{x}}_{0}\right), \hat{\bar{x}}_{0}\right]$, i.e. $R_{0}(x)$. Since we cannot use this residual to reduce the cost of the hedge further, it is tempting to suggest that the optimal solution is given for $\hat{K}\left(\hat{\bar{x}}_{0}\right)=$ $\hat{K}_{0}$, i.e. $\hat{\bar{x}}_{0}=\hat{K}_{0}$ and thus to propose the price bound and robust hedging strategy as presented in proposition 5.1. However, in general the optimum in (56) is achieved for $\hat{K}\left(\hat{\bar{x}}_{0}\right)<\hat{K}_{0}$, i.e. $\hat{\bar{x}}_{0}>\hat{K}_{0}$. This is due to the fact that in order to superreplicate long (short) positions of convex payoffs, the hedging strategy must be set up based on the maximum (minimum) possible volatility. To formalise this, 
Figure 14: Price bounds for different choices of $\bar{x}_{0}$.

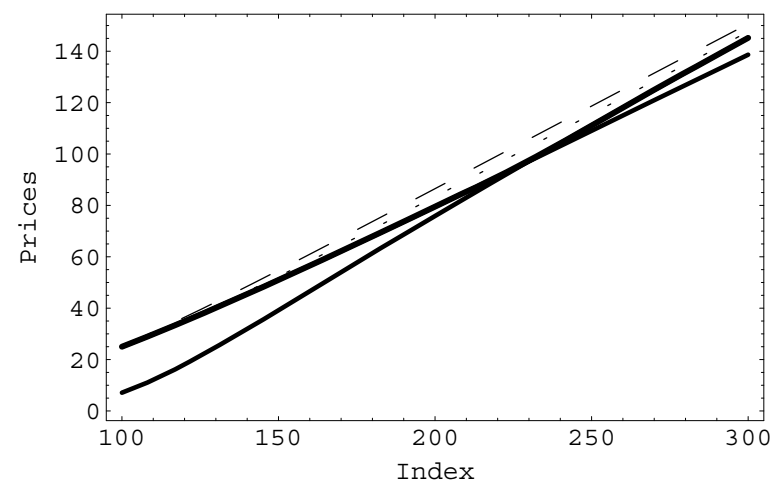

note that

$$
\begin{aligned}
& R_{1}(x)=\left[x-\bar{x}_{0}\right]^{+} f^{\prime}\left(\bar{x}_{0}\right)-\left[f(x)-f\left(\bar{x}_{0}\right)\right]^{+} \\
= & {\left[x-\bar{x}_{0}\right]^{+} f^{\prime}\left(\bar{x}_{0}\right)-\frac{K_{i}}{G\left(t_{i}, t_{N}\right)} } \\
& \times\left[\left(\frac{x G\left(t_{i}, t_{N}\right)}{X_{t_{i}}}\right)^{\alpha}-\left(\frac{\bar{x}_{0} G\left(t_{i}, t_{N}\right)}{X_{t_{i}}}\right)^{\alpha}\right] 1_{\left\{x>\bar{x}_{0}\right\}} \\
= & {\left[x-\bar{x}_{0}\right]^{+} f^{\prime}\left(\bar{x}_{0}\right)-\frac{K_{i} G^{\alpha-1}\left(t_{i}, t_{N}\right)}{X_{t_{i}}^{\alpha}}\left[x^{\alpha}-\left(\bar{x}_{0}\right)^{\alpha}\right]^{+} } \\
= & {\left[x-\bar{x}_{0}\right]^{+} f^{\prime}\left(\bar{x}_{0}\right)-N^{(i)}\left(\alpha, g_{i}\right)\left[x^{\alpha}-\left(\bar{x}_{0}\right)^{\alpha}\right]^{+} }
\end{aligned}
$$

$P\left(\bar{x}_{0}, R_{1}(x), \sigma_{\min }\right)$ is calculated using the lower volatility bound, i.e. we have

$$
\begin{gathered}
P\left(\bar{x}_{0}, R_{1}(x), \sigma_{\min }\right)=f^{\prime}\left(\bar{x}_{0}\right) C\left(X_{t}, \bar{x}_{0}, \sigma_{\min }, t_{N}\right) \\
\quad-N^{(i)}\left(\alpha, g_{i}\right) P O\left(X_{t},\left(\bar{x}_{0}\right)^{\alpha}, \sigma_{\min }, t_{N}\right)
\end{gathered}
$$

where $P O\left(X_{t},\left(\bar{x}_{0}\right)^{\alpha}, \sigma_{\min }, t_{N}\right)$ denotes the price of a power option with power $\alpha$ and strike equal to $\left(\bar{x}_{0}\right)^{\alpha}$ composed at the lower volatility bound, calculated using (7). Finally, with

$$
\begin{aligned}
f^{\prime}\left(\bar{x}_{0}\right) & =\alpha \frac{K_{i} G^{\alpha-1}\left(t_{i}, t_{N}\right)}{X_{t_{i}}^{\alpha}}\left(\bar{x}_{0}\right)^{\alpha-1} \\
& =N^{(i)}\left(\alpha, g_{i}\right)\left(\bar{x}_{0}\right)^{\alpha-1}
\end{aligned}
$$

we get an alternative formulation of (56), i.e. for a given $X_{t}$ we need to solve

$$
\begin{aligned}
& \text { (6o) } \hat{\bar{x}}_{0}=\arg \min _{\bar{x}_{0} \geq \hat{X}_{0}} N^{(i)}\left(\alpha, g_{i}\right)\left[\left(\bar{x}_{0}\right)^{\alpha-1}\right. \\
& \times\left(C\left(X_{t}, \hat{K}\left(\bar{x}_{0}\right), \sigma_{\max }, t_{N}\right)-C\left(X_{t}, \bar{x}_{0}, \sigma_{\min }, t_{N}\right)\right) \\
& \left.+P O\left(X_{t},\left(\bar{x}_{0}\right)^{\alpha}, \sigma_{\min }, t_{N}\right)\right]
\end{aligned}
$$

The minimum is not always achieved for $\hat{K}\left(\bar{x}_{0}\right)=$ $\hat{K}_{0}$, i.e. $\bar{x}_{0}=\hat{K}_{0}$. This is illustrated in Figure 14 .
Here, the dashed line corresponds to the upper price bound, which is obtained for $\bar{x}_{0}=\hat{K}_{0}$, while the dotted line corresponds to the upper price bound, which is obtained for an $\bar{x}_{0}>\hat{K}_{0}$. The thick lines are obtained by inserting solely the upper (lower) volatility bound into (7). In particular, the price bound can be improved in the middle of the range.

Thus, the conservative price bound for the MRRG ${ }^{(i)}$ given in Proposition 5.1 can be improved by using

$$
\begin{aligned}
& \text { Upper price bound at } t=B\left(t, t_{N}\right) \frac{K_{i}}{G\left(t_{i}, t_{N}\right)} \\
& +N^{(i)}\left(\alpha, g_{i}\right)\left[( \hat { \overline { x } } _ { 0 } ) ^ { \alpha - 1 } \left(C\left(X_{t}, \hat{K}\left(\hat{\bar{x}}_{0}\right), \sigma_{\max }, t_{N}\right)\right.\right. \\
& \left.-C\left(X_{t}, \hat{\bar{x}}_{0}, \sigma_{\min }, t_{N}\right)\right) \\
& \left.+P O\left(X_{t},\left(\hat{\bar{x}}_{0}\right)^{\alpha}, \sigma_{\min }, t_{N}\right)\right]
\end{aligned}
$$

where $\hat{\bar{x}}_{0}$ is determined by (6o) (or equivalently (56)). An adjustment of the robust hedging strategy to match the improved upper price bound as well as the an adjustment of the resulting conservative contract parameters is straightforward.

(61) gives the cheapest possible superhedge if (for reasons of tractability) we restrict ourselves to strategies where the payoff of the MRRG is decomposed into convex and concave components, which are then superhedged separately. If one instead superhedges the MRRG in the uncertain volatility model by dynamically switching between $\sigma_{\min }$ and $\sigma_{\max }$ as required by what Avellaneda, Levy and Parás (1995) call the Black/Scholes/Barenblatt (BSB) equation, the initial investment in the hedge (i.e. the price of the superhedge) can be reduced further. However, this entails a considerable loss of tractability, as one must repeatedly solve the BSB equation numerically when searching for a valid zero-premium combination of participation rate and guaranteed return. The superhedge suggested here offers a reasonable compromise between tractability and tightness of the pricing bound.

The cost process $L_{t}^{*}$ corresponding to the superhedge is monotonically decreasing over time $t$, i.e. funds are continually freed from the hedging strategy. Viewed another way, $-L_{t}^{*}$ can be interpreted as the forward value of a bonus account, which accumulates the funds no longer needed to superreplicate the minimum return guarantee. If this surplus is passed on to the insured, the contract value calculated using the upper bound (61) is "fair". 


\section{Conclusion}

The present paper has analysed a minimum-rateof-return guarantee combined with participation in the excess return of a given benchmark portfolio. By casting the embedded option as a power option with power equal to the participation rate, some interesting features are uncovered. For example, in a Black/Scholes-type model framework, an increase in the volatility of the benchmark portfolio does not necessarily lower the annual minimumrate-of-return guarantee, ceteris paribus.

The main focus, however, is on the derivation of meaningful and tractable pricing bounds on the basis of hedging strategies which are robust with respect to uncertain volatility, thus explicitly acknowledging the considerable model risk involved in pricing derivative financial instruments with very long maturities. Basing contract valuation and the determination of fair zero-premium contract parameters on viable hedging strategies recognises that correct pricing alone is insufficient to manage the risk to the insurance (i.e. option) writer.

The fact that the derivative is embedded in an insurance product also means that the "overpricing", which is the necessary consequence of our insistence on a superhedge, is less of an issue here than it would be for a financial derivative offered in a competitive environment. Not only are the potential premia for the former traditionally higher, but also the insurer could mitigate the overpricing by passing funds from the superhedge to the insured as soon as these funds are no longer required, perhaps in a manner analogous to a bonus account of undistributed surplus customary in many life-insurance products. In the extreme this would eliminate "overpricing" altogether.

Thus the analysis of the savings plan presented here can also be seen as of a normative nature. By choosing a combination of guaranteed return and participation rate consistent with the initial investment required for a superhedge and passing on the surplus from the superhedge to the customer at the end of the life of the contract, the arguably best possible combination of two objectives is realised: On the one hand, the embedded option no longer represents an uncontrollable liability to the insurer, while, on the other hand, the hedgeable market risk is eliminated for the insured. The latter only remains exposed, via the uncertainties of the surplus credited to a bonus account, to the unhedgeable risk of uncertain volatility. This is becoming particularly relevant at a time when more and more pension schemes are fully funded through investment portfolios and a properly hedged minimum return guarantee seems politically desirable in order to protect future pensioners from excessive market risk.

\section{Appendix}

\section{A Proof of theorem 2.1}

Notice that the payoff which is directly associated with the premium $K_{i}$ can be represented as

$$
I^{(i)}\left(t_{N}\right):=K_{i} \exp \left\{\bar{g}_{i}\left(t_{N}-t_{i}\right)\right\}
$$

where

$$
\bar{g}_{i}=g_{i}+\frac{\alpha}{t_{N}-t_{i}}\left(\ln \frac{X_{t_{N}}}{X_{t_{i}}}-g_{i}\left(t_{N}-t_{i}\right)\right)^{+}
$$

Defining

$$
G\left(t_{i}, t_{N}\right):=\exp \left\{-g_{i}\left(t_{N}-t_{i}\right)\right\}
$$

we have

$$
\begin{aligned}
& I^{(i)}\left(t_{N}\right)=\frac{K_{i}}{G\left(t_{i}, t_{N}\right)} \\
& \quad \times\left(1+\left[\left(\frac{X_{t_{N}} G\left(t_{i}, t_{N}\right)}{X_{t_{i}}}\right)^{\alpha}-1\right]^{+}\right)
\end{aligned}
$$

Thus, the embedded option is a so-called asymmetric power option (PO), i.e. characterised by the payoff profile

(65) $\left[X_{T}^{\alpha}-K\right]^{+}$.

In the case of the above payoff $I^{(i)}\left(t_{N}\right)$ we have a strike which depends on the power $\alpha$ and the guaranteed minimum rate of return $g_{i}$, i.e.

$$
K^{(i)}\left(\alpha, g_{i}\right):=\left(\frac{G\left(t_{i}, t_{N}\right)}{X_{t_{i}}}\right)^{-\alpha}
$$

The number $N$ of power options embedded into the insurance contract also depends on $\alpha$ and $g_{i}$, i.e.

(67) $N^{(i)}\left(\alpha, g_{i}\right):=K_{i} \frac{G^{\alpha-1}\left(t_{i}, t_{N}\right)}{X_{t_{i}}^{\alpha}}$ 


\section{B Proof of theorem 2.2}

Pricing by no arbitrage implies that the power option price at $t$ is given by

(68) $P O\left(t, X_{t}\right)=B\left(t, t_{N}\right) E_{t_{N}}\left[\left(X_{T}^{\alpha}-K\right)^{+} \mid \mathcal{F}_{t}\right]$

$$
\begin{aligned}
= & B\left(t, t_{N}\right)\left[E_{t_{N}}\left[X_{T}^{\alpha} 1_{\left\{X_{T}^{\alpha}>K\right\}} \mid \mathcal{F}_{t}\right]\right. \\
& \left.-K P_{t_{N}}\left(X_{T}^{\alpha}>K \mid \mathcal{F}_{t}\right)\right]
\end{aligned}
$$

With Equation (6) it follows

$$
\text { (69) } \begin{aligned}
\frac{X\left(t_{N}\right)}{B\left(t_{N}, t_{N}\right)}= & \frac{X(t)}{B\left(t, t_{N}\right)} \\
& \times \exp \left\{-\frac{1}{2} \int_{t}^{t_{N}} \sigma^{2}\left(s, t_{N}\right) d s\right. \\
& \left.+\int_{t}^{t_{N}} \sigma\left(s, t_{N}\right) d W_{t_{N}}(s)\right\}
\end{aligned}
$$

and therefore

(70)

$$
\begin{aligned}
P_{t_{N}} & \left(X_{T}^{\alpha}>K \mid \mathcal{F}_{t}\right)=P_{t_{N}}\left(\alpha \ln X_{T}>\ln K \mid \mathcal{F}_{t}\right) \\
= & P_{t_{N}}\left(\ln \frac{X(t)}{B\left(t, t_{N}\right)}-\frac{1}{2} \int_{t}^{t_{N}} \sigma^{2}\left(s, t_{N}\right) d s\right. \\
& \left.+\int_{t}^{t_{N}} \sigma\left(s, t_{N}\right) d W_{t_{N}}(s)>\frac{1}{\alpha} \ln K \mid \mathcal{F}_{t}\right) \\
= & \mathcal{N}\left(\frac{\ln \frac{X_{t}}{B\left(t, t_{N}\right) \sqrt[a]{K}}-\frac{1}{2} v_{t_{N}^{2}(t)}}{v_{t_{N}}(t)}\right),
\end{aligned}
$$

where

$$
v_{t_{N}}(t)=\sqrt{\int_{t}^{t_{N}} \sigma^{2}\left(s, t_{N}\right) d s}
$$

An application of Ito's lemma gives

$$
\text { (71) } \begin{aligned}
\left(\frac{X\left(t_{N}\right)}{B\left(t_{N}, t_{N}\right)}\right)^{\alpha}=\left(\frac{X(t)}{B\left(t, t_{N}\right)}\right)^{\alpha} \\
\quad \times \exp \left\{-\frac{1}{2} \alpha(1-\alpha) v_{t_{N}}(t)\right\} \frac{D^{t_{N}}\left(t_{N}\right)}{D^{t_{N}}(t)}
\end{aligned}
$$

where

$$
\text { (72) } \begin{aligned}
D^{t_{N}}(t):= & \exp \left\{\alpha \int_{0}^{t} \sigma\left(s, t_{N}\right) d W_{t_{N}}(s)\right. \\
& \left.-\frac{1}{2} \alpha^{2} \int_{0}^{t} \sigma^{2}\left(s, t_{N}\right) d s\right\}
\end{aligned}
$$

such that with $d Q_{t}^{t_{N}}=D_{t}^{t_{N}} d P_{t}^{t_{N}}$ we have

$$
\begin{aligned}
& E_{t_{N}}\left[X_{T}^{\alpha} 1_{\left\{X_{T}^{\alpha}>K\right\}} \mid \mathcal{F}_{t}\right]=\left(\frac{X(t)}{B\left(t, t_{N}\right)}\right)^{\alpha} \\
& \times \exp \left\{-\frac{1}{2} \alpha(1-\alpha) v_{t_{N}}(t)\right\} Q_{t_{N}}\left(X_{T}^{\alpha}>K \mid \mathcal{F}_{t}\right)
\end{aligned}
$$

Finally, using that

(74) $W_{t}^{Q_{t_{N}}}=W_{t}^{P_{t_{N}}}-\alpha \int_{0}^{t} \sigma\left(s, t_{N}\right) d s$

is Brownian Motion with respect to $Q_{t_{N}}$ it follows

$$
\begin{aligned}
& Q_{t_{N}}\left(X_{T}^{\alpha}>K \mid \mathcal{F}_{t}\right)=Q_{t_{N}}\left(\alpha \ln X_{T}>\ln K \mid \mathcal{F}_{t}\right) \\
& =Q_{t_{N}}\left(\ln \frac{X(t)}{B\left(t, t_{N}\right)}-\frac{1}{2} \int_{t}^{t_{N}}\left\|\sigma\left(s, t_{N}\right)\right\|^{2} d s\right. \\
& +\int_{t}^{t_{N}} \sigma\left(s, t_{N}\right)\left(d W_{t_{N}}^{Q}(s)+\alpha \sigma\left(s, t_{N}\right) d s\right) \\
& \left.>\frac{1}{\alpha} \ln K \mid \mathcal{F}_{t}\right) \\
& =\mathcal{N}\left(\frac{\ln \frac{X_{t}}{B\left(t, t_{N}\right) \sqrt[\alpha]{K}}-\frac{1}{2} v_{t_{N}^{2}(t)}}{v_{t_{N}}(t)}+\alpha v_{t_{N}}(t)\right) \\
& =\mathcal{N}\left(\frac{\ln \frac{X_{t}}{B\left(t, t_{N}\right) \sqrt[\alpha]{K}}+\frac{1}{2} v_{t_{N}^{2}(t)}}{v_{t_{N}}(t)}-(1-\alpha) v_{t_{N}}(t)\right)
\end{aligned}
$$

\section{Partial derivatives of the power option pricing formula}

\section{Lemma C.1 Define}

(76) $f(t, z):=z^{\alpha} e^{-\frac{1}{2} \alpha(1-\alpha) v^{2}(t)}$

$$
\begin{aligned}
& \times \mathcal{N}\left(h_{1}\left(t, \frac{z}{\sqrt[a]{K}}\right)-(1-\alpha) v_{t_{N}}(t)\right) \\
& -K \mathcal{N}\left(h_{2}\left(t, \frac{z}{\sqrt[a]{K}}\right)\right),
\end{aligned}
$$

where

$$
\begin{aligned}
& h_{1}(t, z)=\frac{\ln z+\frac{1}{2} v_{t_{N}}^{2}(t)}{v_{t_{N}}(t)} ; \\
& h_{2}(t, z)=h_{1}(t, z)-v_{t_{N}}(t)
\end{aligned}
$$

then it holds

(77) $f_{z}(t, z)=\alpha z^{(\alpha-1)} e^{-\frac{1}{2} \alpha(1-\alpha) v^{2}(t)}$

$$
\times \mathcal{N}\left(h_{1}\left(t, \frac{z}{\sqrt[a]{K}}\right)-(1-\alpha) v_{t_{N}}(t)\right)
$$

(78) $f_{z z}(t, z)=\alpha z^{\alpha-2} e^{-\frac{1}{2} \alpha(1-\alpha) v_{t_{N}}^{2}(t)}$

$$
\begin{aligned}
& \times\left[(\alpha-1) \mathcal{N}\left(h_{1}\left(t, \frac{z}{\sqrt[\alpha]{K}}\right)-(1-\alpha) v_{t_{N}}(t)\right)\right. \\
& \left.+\frac{\mathcal{N}^{\prime}\left(h_{1}\left(t, \frac{z}{\sqrt[a]{K}}\right)-(1-\alpha) v_{t_{N}}(t)\right)}{v_{t_{N}}(t)}\right]
\end{aligned}
$$

(79) $f_{t}(t, z)=v_{t_{N}}(t) v_{t_{N}}^{\prime}(t) z^{2} f_{z z}(t, z)$ 
Proof. Note that

$$
\text { (80) } \begin{aligned}
& \mathcal{N}^{\prime}\left(h_{1}\left(t, \frac{z}{\sqrt[\alpha]{K}}\right)-(1-\alpha) v_{t_{N}}(t)\right) \\
= & \mathcal{N}^{\prime}\left(h_{2}\left(t, \frac{z}{\sqrt[\alpha]{K}}\right)+\alpha v_{t_{N}}(t)\right) \\
= & \frac{1}{\sqrt{2 \pi}} \exp \left\{-\frac{1}{2}\left(h_{2}\left(t, \frac{z}{\sqrt[\alpha]{K}}\right)+\alpha v_{t_{N}}(t)\right)^{2}\right\} \\
= & K z^{-\alpha} e^{\frac{1}{2} \alpha(1-\alpha) v^{2}(t)} \mathcal{N}^{\prime}\left(h_{2}\left(t, \frac{z}{\sqrt[\alpha]{K}}\right)\right.
\end{aligned}
$$

which immediately gives (77). Using (77) we have

$$
\begin{aligned}
& f_{z z}(t, z)=\alpha e^{-\frac{1}{2} \alpha(1-\alpha) v_{t_{N}}^{2}(t)}\left[(\alpha-1) z^{\alpha-2}\right. \\
& \times \mathcal{N}\left(h_{1}\left(t, \frac{z}{\sqrt[\alpha]{K}}\right)-(1-\alpha) v_{t_{N}}(t)\right) \\
& +z^{\alpha-1} \mathcal{N}^{\prime}\left(h_{1}\left(t, \frac{z}{\sqrt[\alpha]{K}}\right)-(1-\alpha) v_{t_{N}}(t)\right) \\
& \left.\quad \times \frac{\partial h_{1}\left(t, \frac{z}{\sqrt[\alpha]{K}}\right)}{\partial z}\right]
\end{aligned}
$$

Notice that with

$$
\begin{aligned}
\frac{\partial h_{1}\left(t, \frac{z}{\sqrt[a]{K}}\right)}{\partial z} & =\frac{\partial}{\partial z}\left(\frac{\ln z-\frac{1}{\alpha} \ln K+\frac{1}{2} v_{t_{N}}^{2}(t)}{v_{t_{N}}(t)}\right) \\
& =\frac{1}{z v_{t_{n}}(t)}
\end{aligned}
$$

we have (78). Finally, it holds

$$
\begin{aligned}
f_{t}(t, z) & =z^{\alpha} e^{-\frac{1}{2} \alpha(1-\alpha) v_{t_{N}}^{2}(t)}\left[-\alpha(1-\alpha) v_{t_{N}}(t)\right. \\
\times & v_{t_{N}}^{\prime}(t) \mathcal{N}\left(h_{1}\left(t, \frac{z}{\sqrt[\alpha]{K}}\right)-(1-\alpha) v_{t_{N}}(t)\right) \\
+ & \mathcal{N}^{\prime}\left(h_{1}\left(t, \frac{z}{\sqrt[\alpha]{K}}\right)-(1-\alpha) v_{t_{N}}(t)\right) \\
\times & \left(h_{t}^{(2)}\left(t, \frac{z}{\sqrt[\alpha]{K}}\right)+\alpha v_{t_{N}}^{\prime}(t)\right) \\
- & \left.K \mathcal{N}^{\prime}\left(h_{2}\left(t, \frac{z}{\sqrt[a]{K}}\right)\right) h_{t}^{(2)}\left(t, \frac{z}{\sqrt[\alpha]{K}}\right)\right]
\end{aligned}
$$

Using (80) gives (79).

\section{Proof of theorem 2.3}

Pricing under No-Arbitrage implies

$$
\begin{aligned}
& \operatorname{MRRG}^{(i)}\left(t, t_{N}\right)=\frac{K_{i} B\left(t, t_{N}\right)}{G\left(t_{i}, t_{N}\right)} \\
& \times\left(1+E_{t_{N}}\left[\left[\left(\frac{X_{t_{N}} G\left(t_{i}, t_{N}\right)}{X_{t_{i}}}\right)^{\alpha}-1\right]^{+} \mid \mathcal{F}_{t}\right]\right) \\
= & \frac{K_{i} B\left(t, t_{N}\right)}{G\left(t_{i}, t_{N}\right)}+N^{(i)}\left(\alpha, g_{i}\right) P O\left(t, X_{t} ; K^{(i)}\left(\alpha, g_{i}\right)\right)
\end{aligned}
$$

where

$$
\text { (85) } \begin{aligned}
K^{(i)}\left(\alpha, g_{i}\right) & :=\left(\frac{G\left(t_{i}, t_{N}\right)}{X_{t_{i}}}\right)^{-\alpha}, \\
N^{(i)}\left(\alpha, g_{i}\right) & :=K_{i} \frac{G^{\alpha-1}\left(t_{i}, t_{N}\right)}{X_{t_{i}}^{\alpha}}
\end{aligned}
$$

with

$$
\frac{X_{t}}{B\left(t, t_{n}\right) \sqrt[\alpha]{K(\alpha, g)}}=\frac{\frac{X_{t}}{X_{t_{i}}}}{\frac{B\left(t, t_{N}\right)}{G\left(t_{i}, t_{N}\right)}}
$$

the above proposition is an immediate consequence of theorem 2.2.

\section{E Proof of theorem 3.2}

For a detailed proof see for example El Karoui, Jeanblanc-Picqué and Shreve (1998) or Dudenhausen, Schlögl and Schlögl (1998). However, the proof can easily be sketched as follows. Assuming lognormal forward index dynamics $X^{*}$ implies in particular that the assumed forward price $\tilde{C}^{*}$ is a function of time and $X^{*}$ such that Itô's lemma gives

(87) $d V^{*}(\Phi)=d \tilde{C}^{*}\left(t, X^{*}\right)$

$$
\begin{aligned}
= & \tilde{C}_{x}^{*}\left(t, X^{*}\right) d X_{t}^{*}+\frac{1}{2} \tilde{C}_{x x}^{*}\left(t, X^{*}\right) d\left\langle X^{*}\right\rangle_{t} \\
& +\tilde{C}_{t}^{*}\left(t, X^{*}\right) d t,
\end{aligned}
$$

i.e. we have

$$
d L_{t}^{*}(\Phi)=\frac{1}{2} \tilde{C}_{x x}^{*}\left(t, X^{*}\right) d\left\langle X^{*}\right\rangle_{t}+\tilde{C}_{t}^{*}\left(t, X^{*}\right) d t
$$

Note that the assumed forward price $\tilde{C}^{*}$ satisfies the Black/Scholes equation, i.e.

$$
\tilde{C}_{t}^{*}(t, z)+\frac{1}{2} z^{2} \tilde{\sigma}^{2}\left(t, t_{N}\right) \tilde{C}_{x x}^{*}(t, z)=0
$$

Using

(90) $\tilde{C}_{t}^{*}\left(t, X^{*}\right)=-\frac{1}{2}\left(X_{t}^{*}\right)^{2} \tilde{\sigma}^{2}\left(t, t_{N}\right) \tilde{C}_{x x}^{*}\left(t, X^{*}\right)$, 
Table 2: Prices and implied volatilities for options on Sydney Futures Exchange SPI 200 stock index futures on 30 March 2001.

\begin{tabular}{|c|c|c|c|c|c|c|c|c|}
\hline Strike & Settlement & Volatility & Strike & Settlement & Volatility & Strike & Settlement & Volatility \\
\hline 2200 & 1061.2 & 23.64 & 2875 & 514.4 & 21.94 & 3550 & 135.1 & 17.35 \\
\hline 2225 & 1038.6 & 23.64 & 2900 & 496.4 & 21.77 & 3575 & 125.9 & 17.18 \\
\hline 2250 & 1016.1 & 23.64 & 2925 & 478.7 & 21.6 & 3600 & 117.1 & 17.01 \\
\hline 2275 & 993.9 & 23.64 & 2950 & 461.3 & 21.43 & 3625 & 108.6 & 16.84 \\
\hline 2300 & 971.8 & 23.64 & 2975 & 444.1 & 21.26 & 3650 & 100.5 & 16.67 \\
\hline 2325 & 950 & 23.64 & 3000 & 427.1 & 21.09 & 3675 & 92.8 & 16.5 \\
\hline 2350 & 928.3 & 23.64 & 3025 & 410.5 & 20.92 & 3700 & 85.5 & 16.33 \\
\hline 2375 & 906.9 & 23.64 & 3050 & 394.1 & 20.75 & 3725 & 78.5 & 16.16 \\
\hline 2400 & 885.7 & 23.64 & 3075 & 378.1 & 20.58 & 3750 & 72 & 15.99 \\
\hline 2425 & 864.8 & 23.64 & 3100 & 362.3 & 20.41 & 3775 & 65.7 & 15.82 \\
\hline 2450 & 844.1 & 23.64 & 3125 & 346.8 & 20.24 & 3800 & 59.8 & 15.65 \\
\hline 2475 & 823.7 & 23.64 & 3150 & 331.7 & 20.07 & 3825 & 54.3 & 15.48 \\
\hline 2500 & 803.5 & 23.64 & 3175 & 316.8 & 19.9 & 3850 & 49.1 & 15.31 \\
\hline 2525 & 783.6 & 23.64 & 3200 & 302.3 & 19.73 & 3875 & 44.3 & 15.14 \\
\hline 2550 & 763.9 & 23.64 & 3225 & 288.1 & 19.56 & 3900 & 41.2 & 15.14 \\
\hline 2575 & 744.5 & 23.64 & 3250 & 274.2 & 19.39 & 3925 & 38.4 & 15.14 \\
\hline 2600 & 725.5 & 23.64 & 3275 & 260.7 & 19.22 & 3950 & 35.7 & 15.14 \\
\hline 2625 & 706.6 & 23.64 & 3300 & 247.5 & 19.05 & 3975 & 33.2 & 15.14 \\
\hline 2650 & 686.5 & 23.47 & 3325 & 234.6 & 18.88 & 4000 & 30.8 & 15.14 \\
\hline 2675 & 666.5 & 23.3 & 3350 & 222.1 & 18.71 & 4025 & 28.6 & 15.14 \\
\hline 2700 & 646.7 & 23.13 & 3375 & 210 & 18.54 & 4050 & 26.5 & 15.14 \\
\hline 2725 & 627.2 & 22.96 & 3400 & 198.2 & 18.37 & 4075 & 24.6 & 15.14 \\
\hline 2750 & 607.8 & 22.79 & 3425 & 186.7 & 18.2 & 4100 & 22.8 & 15.14 \\
\hline 2775 & 588.7 & 22.62 & 3450 & 175.7 & 18.03 & 4125 & 21.1 & 15.14 \\
\hline 2800 & 569.7 & 22.45 & 3475 & 165 & 17.86 & 4150 & 19.6 & 15.14 \\
\hline 2825 & 551.1 & 22.28 & 3500 & 154.6 & 17.69 & 4175 & 18.1 & 15.14 \\
\hline 2850 & 532.6 & 22.11 & 3525 & 144.7 & 17.52 & 4200 & 16.7 & 15.14 \\
\hline
\end{tabular}

The options as well as the underlying futures expired at the end of June 2002. The settlement price of the underlying futures was 3239; the spot price inferred from the immediately expiring futures contract was 3148; the continuously compounded yield for the June 2002 maturity inferred from 90-day bank accepted bills futures also traded on the SFE was 4.7\%. Source: Sydney Futures Exchange.

immediately gives

$$
\text { (91) } \begin{aligned}
d L_{t}^{*}(\Phi)= & \frac{1}{2}\left(X_{t}^{*}\right)^{2} \tilde{C}_{x x}^{*}\left(t, X^{*}\right) \\
& \times\left(\sigma_{t}^{2}\left(T_{N}\right)-\tilde{\sigma}^{2}\left(t, t_{N}\right)\right) d t .
\end{aligned}
$$

\section{F Proof of theorem 5.1}

An easy way to establish an upper price bound is the decomposition of $I^{(i)}\left(t_{N}\right)$ into a linear combination of convex and concave payoffs, i.e.

(92) $I^{(i)}\left(t_{N}\right)=\alpha \frac{K_{i}}{X_{t_{i}}}\left[X_{t_{N}}-\frac{X_{t_{i}}}{G\left(t_{i}, t_{N}\right)}\right]^{+}+M^{(i)}\left(t_{N}\right)$ where

$$
M^{(i)}\left(t_{N}\right)=I^{(i)}\left(t_{N}\right)-\alpha \frac{K_{i}}{X_{t_{i}}}\left[X_{t_{N}}-\frac{X_{t_{i}}}{G\left(t_{i}, t_{N}\right)}\right]^{+}
$$

The first part of the decomposition is equal to the payoff of $\alpha \frac{K_{i}}{\bar{X}_{t_{i}}}$ call options with strike $\hat{K}_{0}=\frac{X_{t_{i}}}{G\left(t_{i}, t_{N}\right)}$ and therefore convex. The upper price bound for this part is thus $\alpha \frac{K_{i}}{\bar{X}_{t_{i}}}$ times the price of the call option composed at the upper volatility bound $\sigma_{\max }$. To see that $M^{(i)}\left(t_{N}\right)$ is indeed concave (and therefore its upper price bound can be calculated using the lower volatility bound $\sigma_{\min }$ ), note that it 
can be represented as

$$
\begin{aligned}
& \frac{K_{i}}{G\left(t_{i}, t_{N}\right)}\left(1+\left[\left(\frac{X_{t_{N}} G\left(t_{i}, t_{N}\right)}{X_{t_{i}}}\right)^{\alpha}\right.\right. \\
& \left.\left.\quad-\alpha \frac{X_{t_{N}} G\left(t_{i}, t_{N}\right)}{X_{t_{i}}}+\alpha-1\right] 1_{\left\{X_{t_{N}}>\frac{X_{t_{i}}}{G\left(t_{i}, t_{N}\right)}\right\}}\right)
\end{aligned}
$$

such that it is enough to show that the function $\psi$ with

(94) $\psi(x):=\left(\frac{x}{b}\right)^{\alpha}-\alpha \frac{x}{b}+\alpha-1$

satisfies the conditions (i) $\psi(b)=0$, (ii) $\psi^{\prime}(x)<0$ for $x>b$ and $\alpha<1$ and (iii) $\psi^{\prime \prime}(x)<0$ for $x>b$ and $\alpha<1$. (i) is trivial, (ii) follows from

$$
\psi^{\prime}(x)=\frac{\alpha x^{\alpha-1}}{b^{\alpha}}-\frac{\alpha}{b}=\frac{\alpha}{b}\left[\left(\frac{b}{x}\right)^{1-\alpha}-1\right]
$$

and (iii) results from

$$
\psi^{\prime \prime}(x)=\frac{\alpha(\alpha-1) x^{\alpha-2}}{b^{\alpha}}=-\frac{\alpha(1-\alpha)}{b^{2}}\left(\frac{x}{b}\right)^{\alpha-2} .
$$

The above proves that a conservative price bound of the MRRG ${ }^{(i)}$ is in fact given by equation (46). Statements (b) and (c) are direct consequences of (a) and (d) follows from Proposition 3.2.

\section{G Market data used in section 4}

Please refer to Table 2.

\section{References}

Avellaneda, Marco, Arnon Levy and Antonio Parás (1995): Pricing and Hedging Derivative Securities in Markets with Uncertain Volatilities, Applied Mathematical Finance, 2 (2): 73-88.

Bacinello, Anna Rita (2001): Fair Pricing of Life Insurance Participating Policies with a Minimum Interest Rate Guaranteed, Astin Bulletin, 31 (2): 275-297.

Bacinello, Anna Rita and Fulvio Ortu (1993): Pricing EquityLinked Life Insurance with Endogenous Minimum Guarantee, Insurance: Mathematics and Economics, 12 (3): 245-258.

Bernard, Carole, Olivier Le Courtois and François QuittardPinon (2005): Market Value of Life Insurance Contracts under Stochastic Interest Rates and Default Risk, Insurance: Mathematics and Economics, 36 (3): 499-516.

Black, Fischer S. and Myron Scholes (1973): The Pricing of Options and Corporate Liabilities, Journal of Political Economy, 81 (3): 637-654.

Bodie, Zvi and Dwight B. Crane (1999): The Design and Production of New Retirement Savings Products, Journal of Portfolio Management, 25 (2): 77-82.
Boyle, Phelim P. and Mary R. Hardy (1997): Reserving for maturity guarantees: Two approaches, Insurance: Mathematics and Economics, 21 (2): 113-127.

Boyle, Phelim P. and Eduardo S. Schwartz (1977): Equilibrium Prices of Guarantees under Equity-Linked Contracts, Journal of Risk and Insurance, 44 (4): 639-660.

Branger, Nicole and Antje B. Mahayni (2006): Tractable Hedging - An Implementation of Robust Hedging Strategies, Journal of Economic Dynamics and Control, 30 (11): 1937-1962.

Brennan, Michael J. and Eduardo S. Schwartz (1976): The Pricing of Equity-Linked Life Insurance Policies with an Asset Value Guarantee, Journal of Financial Economics, 3 (3): 195213.

Brennan, Michael J. and Eduardo S. Schwartz (1979): Alternative Investment Strategies for the Issuers of Equity Linked Life Insurance Policies with an Asset Value Guarantee, Journal of Business, 52 (1): 63-93.

Briys, Eric and François de Varenne (1994): Life Insurance in a Contingent Claim Framework: Pricing and Regulatory Implications, The Geneva Papers on Risk and Insurance Theory, 19 (1): $53-72$.

Briys, Eric and François de Varenne (1997): On the Risk of Insurance Liabilities: Debunking Some Common Pitfalls, The Journal of Risk and Insurance, 64 (4): 673-694.

Chen, An and Michael Suchanecki (2007): Default Risk, Bankruptcy Procedures and the Market Value of Life Insurance Liabilities, Insurance: Mathematics and Economics, 40 (2): 231-255.

Coleman, Thomas, Yohan Kim, Yuying Li and Maria-Cristina Patron (2007): Robustly Hedging Variable Annuities with Guarantees under Jump and Volatility Risks, Journal of Risk and Insurance, 74 (2): 347-376

Dudenhausen, Antje B., Erik Schlögl and Lutz Schlögl (1998): Robustness of Gaussian Hedges under Parameter and Model Misspecification, Technical report, University of Bonn, Department of Statistics.

El Karoui, Nicole, Monique Jeanblanc-Picqué and Steven E. Shreve (1998): Robustness of the Black and Scholes Formula, Mathematical Finance, 8 (2): 93-126.

Esser, Angelika (2003): General Valuation Principles for Arbitrary Payoffs and Applications to Power Options under Stochastic Volatility Models, Financial Markets and Portfolio Management, 17 (3): 351-372.

Geman, Helyette, Nicole El Karoui and Jean-Charles Rochet (1995): Changes of Numeraire, Changes of Probability Measure and Option Pricing, Journal of Applied Probability, 32 (2): 443-458.

Grosen, Anders and Peter L. Jørgensen (2002): Life Insurance Liabilities at Market Value: An Analysis of Insovency Risk, Bonus Policy, and Regulatory Intervention Rules in a Barrier Option Framework, The Journal of Risk and Insurance, 69 (1): 63-91.

Grosen, Anders and Peter L. Jørgensen (1997): Valuation of Early Exercisable Interest Rate Guarantees, Journal of Risk and Insurance, 64 (3): 481-503.

Grosen, Anders and Peter L. Jørgensen (1999): Fair Valuation of Life Insurance Liabilities: The Impact of Interest Guarantees, Surrender Options, and Bonus Policies, Aarhus School of Business, University of Aarhus, working paper.

Harrison, J. Michael and David M. Kreps (1979): Martingales and Arbitrage in Multiperiod Securities Markets, Journal of Economic Theory, 20 (3): 381-408. 
Harrison, J. Michael and Stanley R. Pliska (1981): Martingales and Stochastic Integrals in the Theory of Continuous Trading, Stochastic Processes and their Applications, 11 (3): 215-260.

Heath, David R., Robert A. Jarrow and Aandrew Morton (1992): Bond Pricing and the Term Structure of Interest Rates: A New Methodology for Contingent Claim Valuation, Econometrica, 60 (1): 77-105

Jørgensen, Peter L. (2001): Life Insurance Contracts with Embedded Options, The Journal of Risk Finance, 3 (1): 19-30.

Lyons, Terry J. (1995): Uncertain Volatility and the Risk-free Synthesis of Derivatives, Applied Mathematical Finance, 2 (2): 117-133.

Mahayni, Antje B. and Klaus Sandmann (2006): Return Guarantees with Delayed Payment, forthcoming: German Economic Review.

Miltersen, Kristian R. and Svein-Arne Persson (1998): Guaranteed Investment Contracts: Distributed and Undistributed Excess Return, Odense University, working paper.

Miltersen, Kristian R. and Svein-Arne Persson (2003): Guarantees Investment Contracts: Distributed and Undistributed Excess Return, Scandinavian Actuarial Journal, 2003 (4): 257-279.

Musiela, Marek and Marek Rutkowski (1997): Martingale Methods in Financial Modelling, Vol. 36 of Applications of Mathematics, Springer-Verlag, New York, New York, USA.

Nielsen, Jørgen A. and Klaus Sandmann (1995): Equity-Linked Life Insurance: A Model with Stochastic Interest Rates, Insurance: Mathematics and Economics, 16 (3): 225-253.

Pelsser, Antoon (2002): Pricing and Hedging Guaranteed Annuity Options via Static Option Replication, Erasmus University of Rotterdam, working paper.

Pelsser, Antoon and David F. Schrager (2004): Pricing Rate of Return Guarantees in Regular Premium Unit Linked Insurance, Insurance, Mathematics and Economics, 35 (2): 369-398.

Pooley, David M., Peter A. Forsyth and Kenneth R. Vetzal (2003): Numerical Convergence Properties of Option Pricing PDEs with Uncertain Volatility, IMA Journal of Numerical Analysis, 23 (2): 241-267.

Vanderhoof, Irwin T. and Edward I. Altman (1998): The Fair Value of Insurance Liabilities, The New York University Salomon Center Series on Financial Markets and Institutions, Kluwer Academic Publishers.

Vargiolu, Tiziano (2001): Existence, Uniqueness and Smoothness for the Black-Scholes-Barenblatt Equation, Technical report, Department of Pure and Applied Mathematics, University of Padova.

Zhang, Peter G. (1998): Exotic Options - A Guide to Second Generation Options, World Scientific.

\section{Biographies}

Antje Mahayni, born in 1971, studied economics at the University of Bonn. In 2001, she finished her doctoral theses "Hedging in Incomplete Markets" supervised by Prof. Dr. Dieter Sondermann. From 2001 to 2006 she worked as an Assistant Professor in the finance group of Prof. Dr. Klaus Sandman and received her venia legendi at the University of Bonn with the post-doctoral thesis "Risk Management of Minimum Return Guarantees and Embedded Options." In 2007, Antje Mahayni was appointed to the newly founded chair of "Insurance and Risk Management" at the Mercator School of Management (University of Duisburg-Essen). Her current research interests are placed at the interface of mathematical finance and insurance.

Erik Schlögl is Associate Professor and the Director of the Quantitative Finance Research Centre at the University of Technology, Sydney (UTS). He received his Ph.D. in Economics from the University of Bonn, Germany, in 1997 and has gained broad-based experience in computational financial engineering. In addition to UTS, he held positions at the University of New South Wales and the University of Bonn. He has consulted for financial institutions and software developers in Europe, Australia and in the US. His current research interests focus on credit risk modelling as well as integrating FX and interest rate risk. 